\title{
Globular cluster systems of six shell galaxies ${ }^{\star}$
}

\author{
G. Sikkema ${ }^{1}$, R. F. Peletier ${ }^{1}$, D. Carter ${ }^{2}$, E. A. Valentijn ${ }^{1}$, and M. Balcells ${ }^{3}$ \\ 1 Kapteyn Astronomical Institute, University of Groningen, PO Box 800, 9700 AV Groningen, The Netherlands \\ e-mail: sikkema@astro.rug.nl \\ 2 Astrophysics Research Institute, Liverpool John Moores University, 12 Quays House, Egerton Wharf, Birkenhead, CH41 1LD, UK \\ 3 Instituto de Astrofísica de Canarias, vía Láctea s/n, La Laguna 38200, Spain
}

Received 29 November 2005 / Accepted 14 June 2006

\section{ABSTRACT}

\begin{abstract}
Context. Shells in Elliptical Galaxies are faint, sharp-edged features, believed to provide evidence of a recent $\left(\sim 0.5-2 \times 10^{9}\right.$ years ago) merger event. We analyse the Globular Cluster (GC) systems of six shell elliptical galaxies, to examine the effects of mergers upon the GC formation history.

Aims. We examine the colour distributions, and investigate differences between red and blue globular cluster populations. We present luminosity functions, spatial distributions and specific frequencies $\left(S_{N}\right)$ at $50 \mathrm{kpc}$ radius for our sample.

Methods. We present $V$ and $I$ magnitudes for cluster candidates measured with the HST Advanced Camera for Surveys (ACS). Galaxy background light is modelled and removed, and magnitudes are measured in 8 pixel $(0.4 \operatorname{arcsec})$ diameter apertures. Background contamination is removed using counts from Hubble Deep Field South.

Results. We find that the colour distributions for NGC 3923 and NGC 5982 have a bimodal form typical of bright ellipticals, with peaks near $V-I=0.92 \pm 0.04$ and $V-I=1.18 \pm 0.06$. In NGC 7626, we find in addition a population of abnormally luminous clusters at $M_{I}=-12.5$. In NGC 2865 we find an unusually blue population, which may also be young. In NGC 1344 and NGC 474 the red cluster population is marginally detected. The radial surface density profiles are more flattened than the galaxy light in the cores. As already known, in NGC 3923, which has a high $S_{N}$ of 5.6, the radial density distribution is more shallower than the diffuse galaxy light.

Conclusions. The clusters in NGC 2865 and NGC 7626 provide evidence for formation of a population associated with a recent merger. In the other galaxies, the properties of the clusters are similar to those observed in other, non-shell, elliptical galaxies.
\end{abstract}

Key words. galaxies: peculiar - galaxies: star clusters - galaxies: structure - galaxies: formation - galaxies: evolution

\section{Introduction}

In current galaxy formation models, most ellipticals have already formed at $z>2$ (Ellis et al. 1997; Peebles 2002; van Dokkum et al. 2004). It is unclear whether all globular cluster (GC) systems were also formed at this early epoch or if substantial numbers are still forming today. An important diagnostic is the existence of bimodality in the colour distribution of GCs, present in many early type galaxies (Zepf \& Ashman 1993; Whitmore et al. 1995). Generally this is explained as being due to metallicity differences indicating two or more populations of GCs. Several theories have been proposed to explain the origin of bimodality:

Merger scenarios (Toomre 1977; Schweizer 1987; Ashman \& Zepf 1992) in which the metal-rich GCs were created in gas rich mergers. Since most star formation occurred at early epochs, this means in general that the metal-rich GCs are also old. However, this scenario also suggests that GCs can still be forming today in mergers. This is supported by observations of young cluster-like objects in current mergers in action or possible merger remnants like

^ Based on observations made with the NASA/ESA Hubble Space Telescope, obtained at the Space Telescope Science Institute, which is operated by the Association of Universities for Research in Astronomy, Inc., under NASA contract NAS 5-26555. These observations are associated with program GO9399.
NGC 4038/39 (Whitmore \& Schweizer 1995; Whitmore et al. 1999), NGC 3921 (Schweizer et al. 1996), NGC 7252 (Miller et al. 1997), NGC 1316 (Goudfrooij et al. 2001a,b, 2004), NGC 1700 and NGC 3610 (Whitmore et al. 1997).

Two others models explaining bimodality are the accretion model (Côté et al. 1998) and the multiphase formation model (Forbes et al. 1997; Harris et al. 1998). In both models all GCs are old. The first model produces bimodality by accreting and mixing metal poor GCs from dwarf galaxies with the more metal rich GCs of the massive host galaxy. Cannibalism by our own galaxy of the Sagittarius and Canis Major dwarf galaxies and their clusters (Ibata et al. 1995; Forbes et al. 2004a) and observations of large numbers of dwarf galaxies around giant galaxies are cited as supporting this scenario. The second model explains the bimodality as the result of two phases of GC formation in the initial collapse and formation of a galaxy. The metal poor clusters, and a small proportion of the stars form in the initial gravitational collapse, then the metal rich clusters and the bulk of the stellar component form from enriched gas in a second collapse phase about one or two Gyr later. Strader et al. (2004) argue that this "in situ" model of GC formation is in better agreement with their observations of the correlation of the colours of the metal-poor populations with galaxy luminosity.

A combination of these different scenarios is used in the hierarchical merging model of Beasley et al. (2002), who undertook semi-analytical simulations of GC formation. In this model the metal-poor GCs are old and formed in cold gas 
clumps, the metal-rich ones are created later in merger events. In the hierarchical build up of galaxies, accretion of GCs will also take place. These simulations are able to reproduce the many variations in the colour distributions of GC systems observed in elliptical galaxies as well as the observed $L-N_{\text {tot }}$ relation.

Recently, Yoon et al. (2006), showed that the apparent bimodality in globular cluster colours not necessarily implies a bimodal metallicity distribution. The nonlinear nature of the metallicity-to-colour transformation could cause a single old population with a unimodal metallicity distribution to look bimodal. This model is attractive because it gives a very simple explanation for the observed distributions and could simplify theories of elliptical galaxy formation. However, the observations of recent GC formation, as mentioned above will likely sometimes disturb the predictions made by this model.

There are many examples of multi-colour and spectroscopic data for the GC systems of "normal" elliptical galaxies, and these generally give old ages for both blue and red populations (M 49: Puzia et al. 1999; Cohen et al. 2003; NGC 1399: Forbes et al. 2001b; M 87: Cohen et al. 1998; NGC 1052 and NGC 7332: Forbes et al. 2001a; and in a sample of early-type galaxies: Strader et al. 2005). This is in contrast to studies of the well-known bimodality of the colour distribution of the GCs of the LMC (Gascoigne \& Kron 1952; van den Bergh 1981, 1991) which is primarily an age effect, understood in terms to the evolution with time of clusters in the colour-colour diagram (Frenk \& Fall 1982), possibly combined with a relationship between age and metallicity (Battinelli \& Capuzzo-Dolcetta 1989; Girardi et al. 1995). In ellipticals, the majority of GCs formed at high redshift $(z>2.5)$, whichever formation mechanism is dominant. However it is important to examine evidence that recent merger events can produce enhanced populations of young clusters, as this process may have been much more important in the early universe.

This study focused on a sample of elliptical galaxies with faint stellar shells in their envelopes. Shells and ripples in elliptical galaxies (Malin \& Carter 1980; Malin \& Carter 1983; Schweizer \& Seitzer 1992) are faint, sharp edged stellar features in the envelopes of these galaxies which are the remnants of the stellar components of minor mergers in the comparatively recent history of the galaxy (Quinn 1984). Typical dynamical ages of the shell systems are $\sim 0.5-2 \times 10^{9}$ years (Nulsen 1989; Hernquist \& Quinn 1987). If we can detect a population of clusters then the age of this population will provide an independent estimate of the age since the merger, assuming that the same event produced both the shells and the young clusters. In NGC 2865, one of our sample, Hau et al. (1999) find that the age of a nuclear starburst model for the young stellar population in the core of this galaxy is much older than the dynamical age of the shells, although they do find a better correspondence between ages for a model involving truncation of ongoing star formation. In NGC 1316, Goudfrooij et al. (2001b) find a cluster system with an age of $3.0 \pm 0.5 \mathrm{Gyr}$, consistent with the age of the nuclear stellar population. The shell systems of both NGC 2865 and NGC 1316 are complex, type II or III systems (Prieur 1990) whereas the dynamical age estimates are more directly applicable to simple phase wrapped, type I shell systems.

Two techniques to investigate possible age differences between different GC populations involve measuring radial density profiles, and globular cluster luminosity functions (GCLFs). Radial density profiles generally show a flattening of the globular cluster density profile near the centre, when compared with the profile of the background galaxy light (Lauer \& Kormendy 1987; Capuzzo-Dolcetta \& Donnarumma 2001). Mechanisms which could cause a depletion of the cluster population near the centre are dynamical friction, which causes clusters to spiral in towards the centre (Tremaine et al. 1975; Pesce et al. 1992), and destruction by tidal shocks as the clusters pass close to the nucleus (Ostriker et al. 1989; Capuzzo-Dolcetta \& Tesseri 1997). This second process operates preferentially in triaxial potentials, and on clusters on radial orbits. If we can identify a population of younger clusters, created during a recent merger, then the density profile might extend further into the centre as the clusters have had less time to disrupt. However, this does depend upon the orbital structures to be the same, if one or other population were on predominantly radial orbits, then this would cause a stronger flattening of the core.

The same process can lead to evolution of the mass function of the clusters and hence the GCLF (Fall \& Rees 1977; Gnedin \& Ostriker 1997; Fall \& Zhang 2001). Lower mass clusters are preferentially destroyed, leading to the well known turnover in the GCLF. A younger population would contain more low-mass clusters, and the GCLF should be closer to the original mass function, which might be a power law. Observations of young cluster systems such as NGC 1316 (Goudfrooij et al. 2001b) and NGC 7252 (Miller et al. 1997) do show power law GCLFs.

In this paper we analyse the properties of the GC systems of six shell elliptical galaxies using optical $V$ and $I$ data from the Advanced Camera for Surveys (ACS) on HST. Although the observations were optimised to study the shell structures rather than the GCs, the magnitudes, colours and spatial distribution will indicate whether GC systems of any of the shell galaxies differ from those of normal early type galaxies. We will also provide data on three GC systems which have not been studied before: NGC 474, NGC 1344 and NGC 2865. The last galaxy is known as a recent merger remnant (Hau et al. 1999; Schiminovich 1995). The GC systems of three of our galaxies have been studied before (NGC 3923, ground-based (Zepf et al. 1994, 1995); NGC 5982 and NGC 7626, WFPC2 on the HST (Forbes et al. 1996). The larger field of view and higher resolution of ACS will provide more detections and more complete knowledge of these GC systems.

In Sects. 2 and 3 the observations and data reduction are described, which include the detection and selection of the GCs, calculation of completeness levels and photometry of the globular clusters. Section 4 presents the $V-I$ colour distributions and spatial distributions of the globular cluster systems. Section 5 describes the globular cluster luminosity function which is used in Sect. 6, where the specific frequencies are calculated. Finally in Sects. 7 and 8 we discuss the results and present the conclusions.

\section{Observations and data reduction}

The six shell galaxies (see Table 1) were observed with the ACS_WFC camera between July 2002 and January 2003 with the filters $F 606 W(V)$ and $F 814 W(I)$, with CR_SPLIT $=2$. The ACS camera contains two CCDs of $2048 \times 4096$ pixels, each pixel having a size of $0.049^{\prime \prime}$ pixel $^{-1}$ resulting in a field of view of $202^{\prime \prime} \times 202^{\prime \prime}$. Exposure times were on average $1000 \mathrm{~s}$, see Table 2. The inner 8 pixels of NGC 474 and the inner 24 pixels of NGC 2865 were saturated in both $V$ and $I$.

Standard reduction was carried out in the IRAF+STSDAS ${ }^{1}$ environment, using the packages CALACS and PyDrizzle.

1 IRAF is distributed by the National Optical Astronomy Observatories, which are operated by the Association of Universities for Research in Astronomy, Inc., under cooperative agreement with the National Science Foundation. 
Table 1. Properties of six shell galaxies. Data in Cols. 2-9 from Roberts (1991). 1st column: Galaxy name; 2nd and 3rd columns: Right Ascension and Declination; 4th and 5th columns: galactic longitude and latitude; 6th and 7th columns: extinction coefficients in $V$ and $I$ magnitudes from Schlegel et al. (1998); 8th column: morphological type (throughout this paper we assumed that NGC 474 is elliptical and not S0 (Hau et al. 1996); 9th column: total apparent $V$ magnitude; 10th column: SBF distance modulus in $I$, corrected for extinction (Tonry et al. 2001). 11th column: distances adopted in this paper using Col. 10 except $\$$ : GCLF distances for NGC 1344 and NGC 3923, calculated in Sect. 5.2.; 12th column: $D n-\sigma$ distances from Faber et al. (1989); 13th column: central velocity dispersion $\sigma$; typical errors are $10 \mathrm{~km} \mathrm{~s}^{-1}$ (HYPERLEDA ${ }^{4}$ ).

\begin{tabular}{|c|c|c|c|c|c|c|c|c|c|c|c|c|}
\hline Galaxy & RA (J2000) & $\operatorname{Dec}(\mathbf{J} 2000)$ & (4) & $\begin{array}{r}b \\
(5) \\
\end{array}$ & $\begin{array}{r}A_{V} \\
\mathrm{mag} \\
(6) \\
\end{array}$ & $\begin{array}{r}A_{I} \\
\mathrm{mag} \\
(7) \\
\end{array}$ & $\begin{array}{r}\text { type } \\
(8) \\
\end{array}$ & $\begin{array}{r}m_{V} \\
\operatorname{mag} \\
(9) \\
\end{array}$ & $\begin{array}{l}m-M \\
\text { mag } \\
(10) \\
\end{array}$ & $\begin{array}{l}d \\
\mathrm{Mpc} \\
(11) \\
\end{array}$ & $\begin{array}{l}D n-\sigma \\
\mathrm{Mpc} \\
(12)\end{array}$ & $\begin{array}{l}\sigma \\
\mathrm{km} \mathrm{s}^{-1} \\
(13) \\
\end{array}$ \\
\hline NGC 474 & $1^{\mathrm{h}} 20^{\mathrm{m}} 06^{\mathrm{s}} .7$ & $03^{\circ} 24^{\prime} 55^{\prime \prime}$ & 136.80 & 58.68 & 0.11 & 0.07 & E? & 11.39 & $32.56 \dagger$ & 32.5 & - & 164 \\
\hline NGC 1344 & $3^{\mathrm{h}} 28^{\mathrm{m}} 19.7$ & $-31^{\circ} 04^{\prime} 05^{\prime \prime}$ & 229.07 & -55.68 & 0.06 & 0.04 & E5 & 10.41 & 31.48 & $22.1 \div$ & 20 & 187 \\
\hline NGC 2865 & $9^{\mathrm{h}} 23^{\mathrm{m}} 30^{\mathrm{s}} .2$ & $-23^{\circ} 09^{\prime} 41^{\prime \prime}$ & 252.95 & +18.94 & 0.27 & 0.16 & E3-4 & 11.30 & 32.89 & 37.8 & $25 \diamond$ & $230 \diamond$ \\
\hline NGC 3923 & $11^{\mathrm{h}} 51^{\mathrm{m}} 01^{\mathrm{s}} .8$ & $-28^{\circ} 48^{\prime} 22^{\prime \prime}$ & 286.53 & +33.32 & 0.27 & 0.16 & E4-5 & 9.88 & 31.80 & $20.0 \ddagger$ & 21 & 249 \\
\hline NGC 5982 & $15^{\mathrm{h}} 38^{\mathrm{m}} 39^{\mathrm{s}} .8$ & $+59^{\circ} 21^{\prime} 21^{\prime \prime}$ & 93.10 & +46.92 & 0.06 & 0.04 & E3 & 11.20 & $33.11 \dagger$ & 41.9 & 41 & 240 \\
\hline NGC 7626 & $23^{\mathrm{h}} 20^{\mathrm{m}} 42^{\mathrm{s}} \cdot 3$ & $+08^{\circ} 13^{\prime} 02^{\prime \prime}$ & 87.86 & -48.38 & 0.24 & 0.14 & Epec & 11.25 & $33.41 \dagger$ & 48.2 & 46 & 270 \\
\hline
\end{tabular}

$\dagger$ : Distance moduli taken from Roberts et al. (1991) determined from HI velocity data, corrected for galactic rotation and restframe of the Local Group using $H_{0}=75 \mathrm{~km} \mathrm{~s}^{-1} \mathrm{Mpc}^{-1} . \diamond: d(D n-\sigma)$ for NGC 2865 is probably wrong, since NGC 2865 exhibits a central depression in $\sigma$ due to a rotating disk (Hau et al. 1999). Extrapolating his data gives $\sigma=230 \mathrm{~km} \mathrm{~s}^{-1}(d(D n-\sigma)=34.7 \mathrm{Mpc})$, much larger than the value used by Faber et al. $\left(\sigma=168 \mathrm{~km} \mathrm{~s}^{-1}\right)$.

Table 2. Observational characteristics. 1st column: Galaxy name; 2 st and 3nd columns: $V$ and $I$ exposure times in seconds; 4th and 5th columns: $80 \%$ completeness levels in $V$ and $I$; 6th and 7th columns: adopted background value in counts.

\begin{tabular}{lcccccc}
\hline \hline$(1)$ & $(2)$ & $(3)$ & $(4)$ & $(5)$ & $(6)$ & $(7)$ \\
Galaxy & exp. $V(\mathrm{~s})$ & exp. $I(\mathrm{~s})$ & $80 \% V(\mathrm{mag})$ & $80 \% I(\mathrm{mag})$ & bg. $V(\mathrm{cnts})$ & bg. $I(\mathrm{cnts})$ \\
\hline NGC 474 & 1140 & 960 & 25.74 & 24.78 & 203 & 129 \\
NGC 1344 & 1062 & 840 & 25.81 & 24.76 & 94 & 47 \\
NGC 2865 & 1020 & 840 & 25.85 & 24.78 & 146 & 109 \\
NGC 3923 & 1140 & 978 & 25.59 & 24.47 & 170 & 103 \\
NGC 5982 & 1314 & 1020 & 26.08 & 24.80 & 124 & 182 \\
NGC 7626 & 1140 & 960 & 25.96 & 24.86 & 119 & 80 \\
\hline
\end{tabular}

These are provided by the Space Telescope Science Institute (STScI). CALACS processing includes bias and dark subtraction, removal of the overscan regions, flat fielding and cosmic ray rejection. The default pipeline is not efficient at removing cosmic rays when the image is filled by a large galaxy. To solve this problem we changed the value of CALACS pipeline parameter SCALENSE from 0.3 to 0.0. Setting SCALENSE to 0.0 increases the probability of removing good stellar data ${ }^{2}$. However, this is only true for empty fields, which is not the case for our data. The resulting images were further processed by PyDrizzle, which removes the geometric distortion of the ACS optical configuration. Finally, after drizzling the images, the IRAF package LA_COSMIC (van Dokkum 2001) was used to remove any remaining cosmic rays, which still affected several hundreds of pixels in each image. The standard ACS photometric calibration was used (Sirianni et al. 2005) to obtain Johnson $V$ and Cousins $I$ magnitudes. The following transformation formulae were applied:

$V_{J}=m(F 606 W)+26.331+0.340 \times(V-I)_{J C}-0.038 \times(V-I)_{J C}^{2}(1)$

$I_{C}=m(F 814 W)+25.496-0.014 \times(V-I)_{J C}+0.015 \times(V-I)_{J C}^{2} \cdot(2)$

Here $m(F 606 W)$ and $m(F 814 W)$ are ACS Vega instrumental magnitudes and $V$ and $I$ are in Johnson and Cousins systems respectively. The Johnson and Cousins magnitudes were corrected

\footnotetext{
${ }^{2}$ http://www.stsci.edu/hst/acs/documents/ newsletters/stan0301.html
}

for galactic extinction using the values of Schlegel et al. (1998), which are listed in Table 1.

\section{Data analysis}

In this section we describe how the globular cluster source catalogues were obtained and describe their characteristics in terms of photometric errors and completeness, which are used in the further analysis of the data.

\subsection{GALPHOT}

Information about the morphology of the galaxies was obtained by using the ellipse fitting task GALPHOT (see Jørgensen et al. 1992); it returns information such as ellipticity, position angle, surface brightness and the C3, C4, S3, S4 coefficients (Carter 1978), all as a function of radius. A galaxy subtracted residual image is also returned, which we used to extract the GCs.

In the GALPHOT processing, background galaxies, pointlike objects, dust lanes, bright pronounced shells and additional bad data were masked out in an iterative way. Remaining faint shell structures, having a brightness typically not more than $5 \%$ of the galaxy light, do not severely affect the final results. The best fits were obtained by allowing the center, position angle and ellipticity to be free parameters. In two cases: NGC 2865 and NGC 5982 the central regions could not be subtracted in a proper way, these regions correspond to circles with diameters 4 " for 
NGC 2865 and 24" for NGC 5982 respectively. Note that the inner $0.3^{\prime \prime}$ is saturated in the center of the latter galaxy.

Light profiles were obtained by plotting the surface brightness for each fitted ellipse as function of radius. The outer parts of the light profiles are severely affected by uncertainty in the determination of the background. It is difficult to determine a reliable background values from the ACS images themselves, since the galaxies fill the whole field. Fortunately, for five galaxies we found optical wide field data in the $R$ band in the ESO archive of WFI@2 . 2m. The WFI camera has a field of view of $34^{\prime} \times 33^{\prime}$, much larger than the galaxies.

We used the ASTRO-WISE system ${ }^{3}$ (Valentijn \& Kuijken 2004) to reduce the WFI images. After subtracting a constant background value from the WFI images, GALPHOT was applied to them. The ACS background values were determined by matching ACS light profiles to the WFI light profiles. For the galaxy without WFI data, NGC 5982, we extrapolated from the outer points of the image using a de Vaucouleurs (1948) $r^{1 / 4}$ law. The calculated values for the backgrounds are listed in Table 2. The final GALPHOT results describing the morphology of the six galaxies will be presented in a future paper (Sikkema et al. in preparation).

\subsection{Globular cluster candidates}

The galaxy subtracted, residual images were used for source detection and photometry of globular cluster candidates (GCCs). SExtractor (Bertin \& Arnouts 1996) was used for this purpose. The keyword BACK_FILTERSIZE was set to 5; the keyword BACK_SIZE was set to the relatively small value of 24 , to account for background variations caused by broad shell regions, diffracted light from bright stars, and dusty regions.

\subsection{Photometry}

Because we are analysing point like objects, the magnitudes are measured in fixed apertures, and we tested apertures with diameters of $4,6,8,10,12,14,16,18,20$ pixels. The quality of the photometry was analysed by adding objects to the residual images using the IRAF task MKOBJECT (the object was extracted from an ACS image and is a typical PSF object with a few thousands counts at its peak). We did this 5 times, each time introducing 350 objects with a uniform magnitude distribution and positions (generated by IRAF/STARLIST. Catalogues were extracted by using SExtractor again with the same parameters as before. The resulting catalogues in $V$ and $I$ were then associated, keeping only associations within positional error ellipses of $1 \times F W H M=2.4$ pixels. Comparing input STARLIST data with output data gives information about photometric errors and completeness levels.

\subsection{Completeness}

We define completeness as the ratio of recovered objects divided by the original number of added objects measured in a particular magnitude bin. Figure 1 shows a typical result of this procedure for the $I$ band residual image of NGC 3923. On the horizontal axis are the input STARLIST magnitudes. The completeness is plotted on the vertical axis. The horizontal lines indicate $50 \%$ and $80 \%$ completeness levels. The results for each image are shown in Table 2.

\footnotetext{
${ }^{3}$ www.astro-wise.org/portal
}

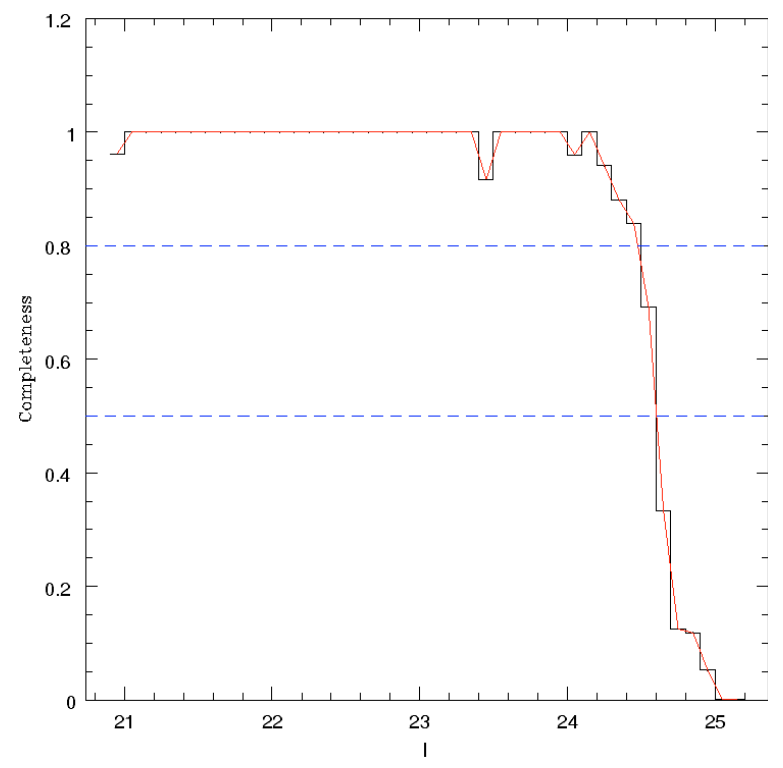

Fig. 1. Histogram completeness for NGC 3923 I band image, using the gc-type object. Vertically: completeness ratio; horizontal scale: instrumental ACS magnitudes. Dashed lines indicate $80 \%$ and 50\% completeness levels.

\subsection{Photometric errors and aperture selection}

The photometric errors were obtained by subtracting input STARLIST magnitudes from output aperture magnitudes, where extreme outliers (mainly due to false associations) in each magnitude bin were removed using $5 \times$ sigma clipping. Typical results, using the NGC 3923 I band residual image and an 8 pixel aperture, are shown in Fig. 2. The upper panel shows rms errors in magnitudes in each bin; off-sets from STARLIST magnitudes are plotted in the lower panel. The magnitude errors depend on the different apertures in various ways:

- the rms-errors at the $80 \%$ completeness levels increase for larger apertures: from 0.08 mag using a 4 pixel aperture, to 0.18 mag using a 20 pixel aperture;

- the measured magnitudes show systematic off-sets for each aperture; larger apertures give smaller offsets. We assume that the offsets of the real data are the same in all of our twelve images;

- the off-sets vary within each magnitude bin; however the variations are larger for large apertures.

Considering these three error sources leads to a optimum aperture diameter of 8 pixels, which was used in all remaining analysis. The photometric error is $\approx 0.10 \mathrm{mag}$. near the $80 \%$ completeness levels and the aperture correction is 0.26 mag. Figure 2 shows an example using this aperture for the NGC 3923 I band residual image.

Before doing the photometry, we checked whether globular clusters are resolved in our galaxies. Table 1 shows that our closest galaxies, NGC 1344 and NGC 3923, have a distance of about $20 \mathrm{Mpc}$. At this distance, typical galactic globular clusters, which have a half total light diameter of $6 \mathrm{pc}$ (van den Bergh 1994), will have an angular size of somewhat more than 1 ACS pixel. We conclude that most globular clusters will be unresolved.

\footnotetext{
${ }^{4}$ http://www-obs. univ-lyon1.fr/hypercat
} 


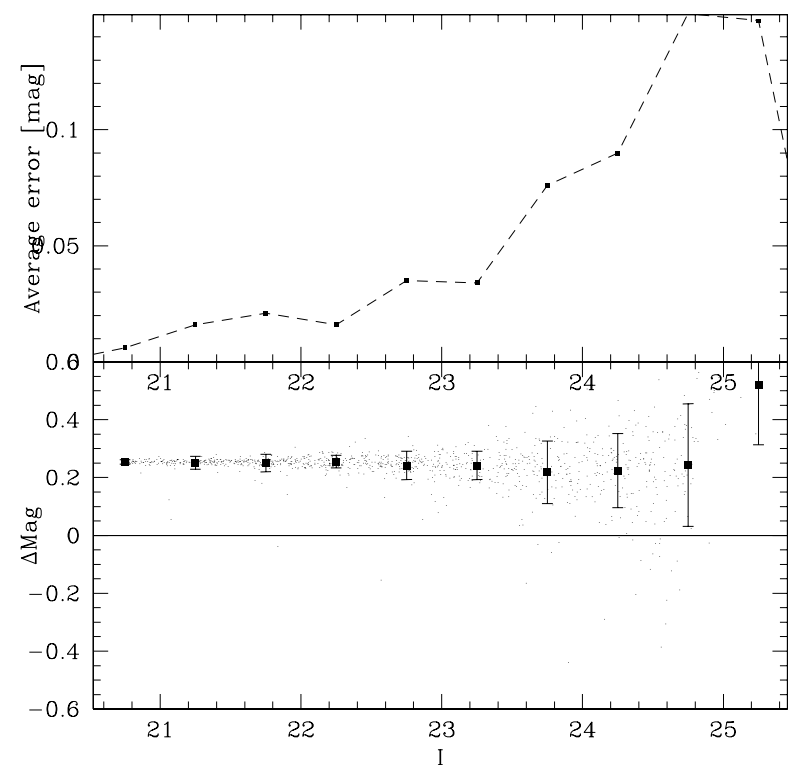

Fig. 2. Photometric errors for artificial pointlike objects in the GALPHOT NGC 3923 I band residual image. Top: rms errors in magnitudes in each bin; bottom: off-sets from STARLIST artificial objects using an aperture of 8 pixels.

\subsection{Selection of GCCs}

We made use of various SExtractor keywords to select the globular cluster candidates. We kept all objects which have ELONGATION $<1.4$ (keep round looking objects), $1.8<$ FWHM_IMAGE $<5$ (removing most spurious objects and extended objects) and FLAGS $>0$ (removing objects which are blended, saturated or placed near other bad pixels). Objects fainter than the $80 \%$ completeness levels were also removed. The remaining lists of objects in $V$ and $I$ were associated using positional error ellipses of $1 \times F W H M=2.4$ pixels, which again removed many objects. The final source list was further cleaned by visual inspection of the residual images, thereby removing any false data, for instance bright galaxy cores, sources located near borders or detections in spikes of bright stars. Figure 3 shows the distribution of the remaining objects.

Before using these data, we note that the source catalogue is still contaminated by two sources: foreground stars and background galaxies. Below, an estimate of these numbers is made.

1. Galactic foreground stars. The number of foreground stars depends upon galactic coordinates. Table 1 shows that NGC 2865, with the lowest galactic latitude of $19^{\circ}$, is likely to be affected most by foreground stars. We estimated the number of stars in each field by using The Besançon model (Robin et al. 2003). We used the following input for the model:

- an error polynomial $=$ a polynomial was fitted to our rmserror curve (top panel in Fig. 2) and given as input;

- magnitude limits $=$ our $80 \%$ completeness levels;

- field of view = ACS field size;

- galactic coordinates.

The model returns catalogues with the expected number of stars and their $V$ and $I$ magnitudes. Generally we find that the number of stars is negligible compared with the number of GCs. A more detailed discussion of the effect of this contaminant is given in Sect. 4.1.

2. Unresolved background galaxies. An estimate of the number of these contaminants was made by using the Hubble Deep

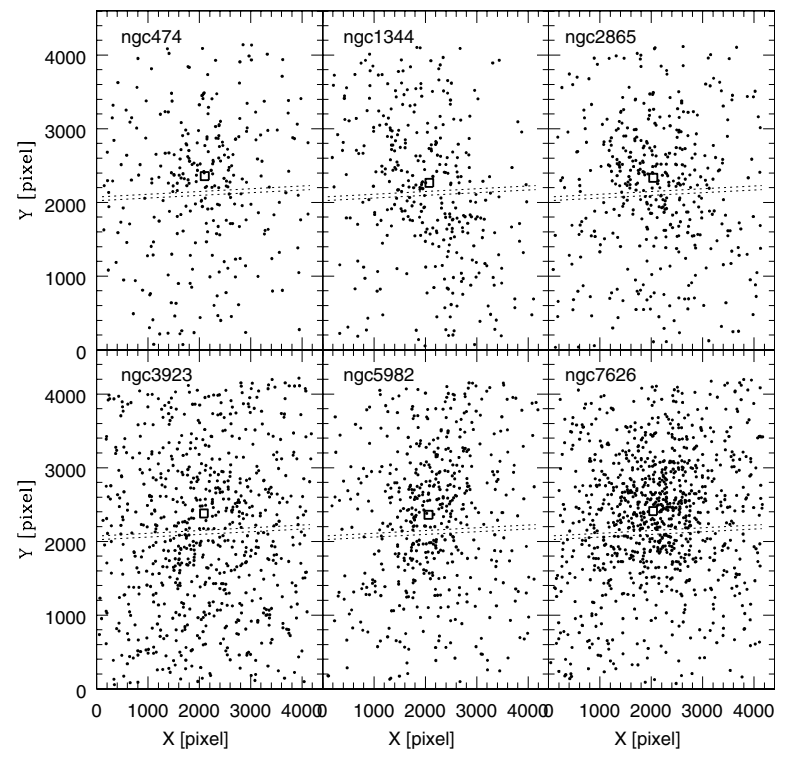

Fig. 3. Location of globular clusters, using ACS pixel coordinates (pixelsize $=0.05$ arcsec; the open square represents the center of the galaxy.

Field South (Williams et al. 1996). The images are publicly available and were observed in the same passbands as our data. Since the HDFS images are much deeper than our images, we dimmed the HDFS in order to reach our $80 \%$ completeness levels. We did this by dimming the HDFS $V$ and $I$ images with 2.8 and 2.6 magnitudes respectively, by multiplying with $10^{-\langle\Delta \mathrm{mag}\rangle / 2.5}$. To restore the noise levels of the original images, we added Gaussian noise. Next, SExtractor was applied with the same parameters as before, selecting objects using the same selection criteria as before and then associating the objects. We find 15 sources within $0.55<V-I<1.45$, which are evenly spread in $V-I$. This number, together with the number of foreground stars, will be used in Sect. 4.4 to estimate a contaminating background density valid for each galaxy.

\section{4. $V-I$ distributions and spatial distributions}

\subsection{V-I distributions}

To appear in our histograms an object must be detected in both passbands, so to avoid colour bias in the selection, we selected only objects from our source catalogue which are $0.3 \mathrm{mag}$ brighter in both $V$ and $I$ than the respective $80 \%$ completeness limits. Figure 1 shows that we reach $100 \%$ completeness at these brighter magnitude limits. Another advantage of doing this, is that the photometric errors are smaller at these limiting magnitudes $(0.07$ mag instead of 0.10$)$. The final $V-I$ colour distributions are depicted in the panels of Fig. 4. The upper part of each panel shows the distribution of objects in the colour-absolute magnitude plane. Here, the vertical axis is absolute $I$-band magnitude, which was determined using the distances listed in Col. 11 of Table 1 . The lower part shows $V-I$ histograms with binsize $0.075 \mathrm{mag}$. In these panels the upper, thin-line histogram represents the data, the lower, thick-line histogram represents the residuals from the fits described in Sect. 4.2.

The expected number of stars in the Besançon catalogues is very low compared to the number of GCCs and will not influence any conclusion drawn from the figures. In the NGC 2865 data, the galaxy at the lowest galactic latitude, there is a small bump 


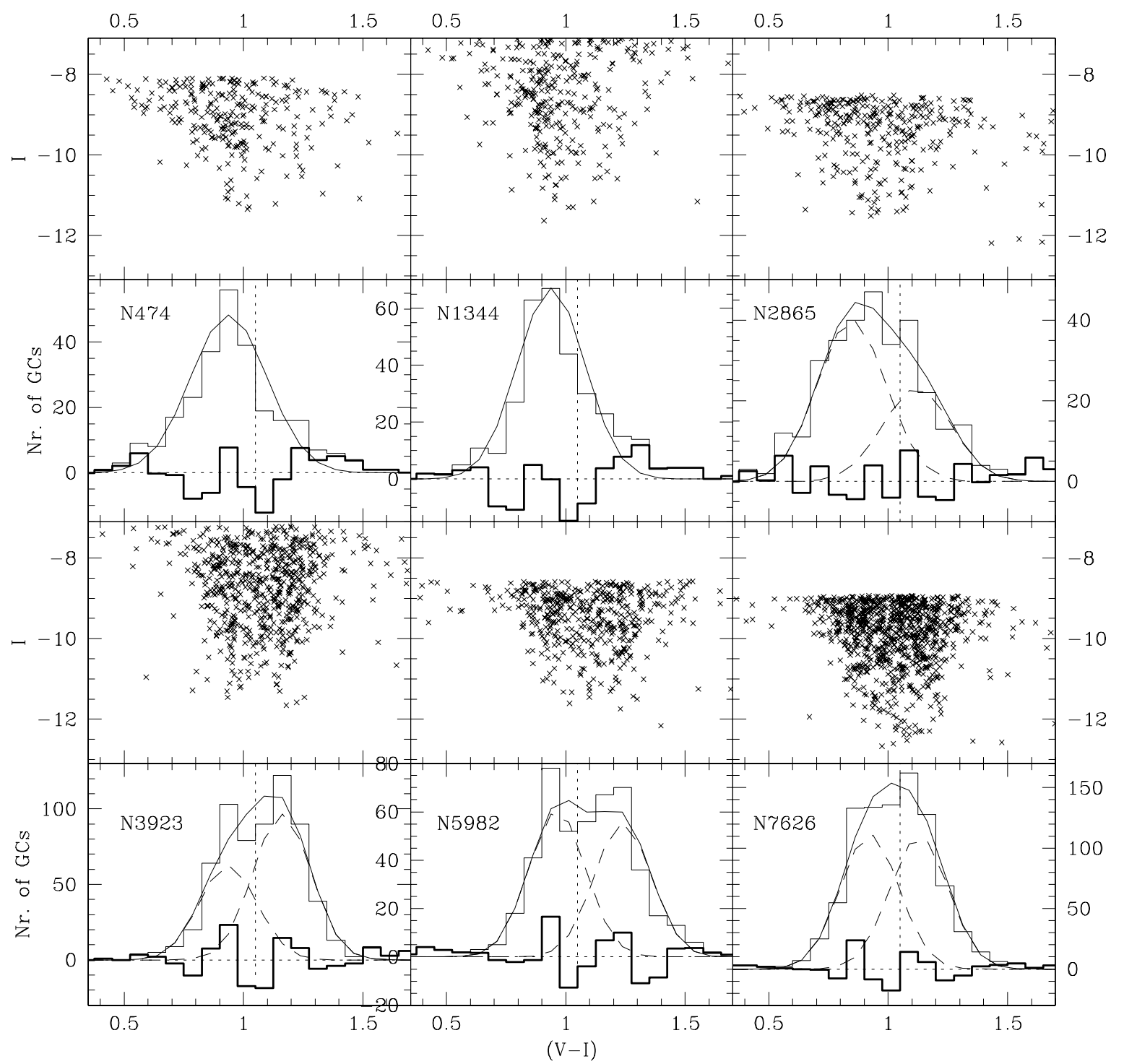

Fig. 4. Histograms and colour diagram of $V-I$ colours of the globular cluster candidates. All histograms are better fit by two Gaussians than one, but for NGC 1344 and NGC 474 we plot only the blue component as a Gaussian fit, as the numbers in the red peak are not significant. For the other four galaxies we plot the two Gaussians fit by the KMM algorithm. Residual histograms (thick lines) represent the difference between the data and the sum of the Gaussian fits. Vertical, dashed lines represent the separation of the data into red and blue groups at $(V-I)=1.05$.

near $V-I=1.6$, which is also expected from the Besançon model. The curves and thick lines are Gaussian fits to the histograms and their residuals respectively (see Sect. 4.2).

\subsection{Components of the colour distributions}

Inspection of the histograms in Fig. 4 suggests that the colour distributions of GCs for NGC 3923 and NGC 5982 have the bimodal form which is normal for bright ellipticals, with blue and red peaks near $(V-I)=0.92$ and $(V-I)=1.18$ respectively. This bimodal form is parameterised conventionally as the sum of two Gaussians. In Sect. 7 we investigate the ways in which the other four histograms differ from this form.

A check for colour bimodality was made by applying the KMM algorithm (Ashman et al. 1994) and DIP test (Gebhardt \& Kissler-Patig 1999) on data points between $0.55<$ $V-I<1.45$. KMM was used in standard mode (fitting two Gaussians with equal $\sigma$ 's). It returns the value $P$, which indicates if a distribution is better characterised by a sum of the two
Gaussians than a single Gaussian. Table 3 lists the output which consists of peak values, number counts in each Gaussian and the $P$-value.

The KMM analysis shows that all of the colour distributions are better fit by a double than a single Gaussian, but in NGC 1344 and NGC 474 the numbers of clusters in the red (metal-rich) Gaussian are too small to be statistically significant, so we plot in Fig. 4 only the blue Gaussian for these two galaxies. For NGC 7626 and NGC 2865 neither a single nor double Gaussian provides a good fit to the histogram, and the structure is more complex. However we do show in Fig. 4 the blue and red Gaussians generated by the KMM algorithm.

The obvious colour bimodality for NGC 3923 and NGC 5982 is confirmed by using the DIP test (Gebhardt \& Kissler-Patig 1999). This test calculates the probability of a dip occurring in a supposedly bimodal distribution. Applying this test to the colour distributions of our six galaxies gives significant bimodality for NGC 3923 and NGC 5982 with the dip probability $P_{\text {dip }}=0.99$ and $P_{\text {dip }}=0.92$ respectively. 
Table 3. Left: output of KMM algorithm (explanation: see Sect. 4.2) with blue and red $V-I$ peaks (Cols. 2, 3), KMM assignments of GCs to blue and red groups (Cols. 4, 5) and $P$-value (Col. 6), see also Fig. 4. Right: slopes $\alpha$ and errors of the surface densities of red, blue (based on a split at $V-I=1.05$ ) and all GCs (Cols. 8-13), determined applying a weighted least squares method on ranges listed in Col. 7. Last column: slope of galaxy light. Surface densities of all, red and blue groups are shown in Figs. 8-10.

\begin{tabular}{|c|c|c|c|c|c|c|c|c|c|c|c|c|c|c|}
\hline $\begin{array}{l}\text { Galaxy } \\
\text { (1) }\end{array}$ & $\begin{array}{r}\mu_{\text {blue }} \\
(2)\end{array}$ & $\begin{array}{r}\mu_{\text {red }} \\
(3)\end{array}$ & $\begin{array}{r}\text { ass. bl. } \\
\text { (4) }\end{array}$ & $\begin{array}{r}\text { ass. rd. } \\
\text { (5) }\end{array}$ & $\begin{array}{r}P \text {-value } \\
(6)\end{array}$ & $\begin{array}{r}\log (r) \\
(7)\end{array}$ & $\begin{array}{r}\alpha_{\text {red }} \\
(8) \\
\end{array}$ & $\begin{array}{l}\text { err } \\
\text { (9) }\end{array}$ & $\begin{array}{r}\alpha_{\text {blue }} \\
(10)\end{array}$ & $\begin{array}{r}\text { err } \\
(11)\end{array}$ & $\begin{array}{r}\alpha_{\text {all }} \\
(12)\end{array}$ & $\begin{array}{r}\text { err } \\
(13)\end{array}$ & $\begin{array}{r}\alpha_{\text {galaxy }} \\
(14)\end{array}$ & $\begin{array}{r}\text { err } \\
(15)\end{array}$ \\
\hline NGC 474 & 0.90 & 1.22 & 224 & 34 & 0.035 & $1.2-2.2$ & - & - & - & - & -1.50 & 0.14 & -1.87 & 0.03 \\
\hline NGC 1344 & 0.92 & 1.21 & 268 & 49 & 0.004 & $1.5-2.2$ & - & - & - & - & -2.02 & 0.17 & -2.23 & 0.02 \\
\hline NGC 2865 & 0.85 & 1.12 & 192 & 111 & 0.039 & $1.35-2.15$ & -3.05 & 0.24 & -2.04 & 0.17 & -2.28 & 0.14 & -2.04 & 0.01 \\
\hline NGC 3923 & 0.94 & 1.16 & 253 & 390 & 0.036 & $1.5-2.1$ & -0.87 & 0.15 & -0.86 & 0.15 & -0.90 & 0.10 & -1 & 0.03 \\
\hline NGC 5982 & 0.96 & 1.24 & 240 & 221 & 0.000 & $1.4-2.1$ & -1.98 & 0.18 & -1.22 & 0.19 & -1.68 & 0.13 & -2.23 & 0.04 \\
\hline NGC 7626 & 0.92 & 1.13 & 455 & 448 & 0.039 & $1.5-2.2$ & -1.99 & 0.14 & -1.42 & 0.13 & -1.67 & 0.09 & -2.16 & 0.04 \\
\hline
\end{tabular}

NGC 3923 and NGC 5982 have been studied before. NGC 3923 has been observed from the ground in the Washington system (Zepf et al. 1995). Like us, they found a bimodal distribution, which, converting their data into $V-I$ (Forbes \& Forte 2001) and correcting to our extinction scale, give colours of $1.01( \pm 0.05)$ and $1.21( \pm 0.05)$, an offset of +0.06 with respect to our data. Data on NGC 5982 (Kundu \& Whitmore 2001a) also revealed a bimodal distribution; applying our extinction scale to their data gives $V-I$ colours of $0.96( \pm 0.03)$ and $1.15( \pm 0.03)$. While the blue peak is the same as ours, their red peak is 0.1 magnitude bluer. We attribute this to our much larger GC sample.

In the further analysis we distinguish between red and blue sample by cutting the distribution at $(V-I)=1.05$, in order to analyse whether these two populations have different properties.

If we fit the $V-I$ histogram of NGC 2865 with 2 Gaussians, as we do for NGC 3923, NGC 5982 and NGC 7626, the blue peak extends much further to the blue than in other galaxies. We suggest in Sect. 7 that this can be attributed due to a population of young blue clusters, overlapping in colour with the normal old metal-poor population.

For NGC 7626 KMM gives maxima at 0.92 and 1.13; the bimodal nature was already observed by earlier, but less deep, observations (Kundu \& Whitmore 2001a). However inspection and the output of KMM show that the simple bimodal form is not a good fit, instead the histogram shows a broad flat peak. The middle of this peak $((V-I) \sim 1.0)$ is filled by exceptionally bright $\left(M_{I}<-11\right)$ and probably young GCs (see Sect. 7.3). The colour magnitude diagram shows that these are distributed in several small clumps in, which cannot be explained by random effects: the errors in $V-I$ at these magnitudes $\left(m_{V} \approx 23.0\right)$ are at most 0.04 (Fig. 2) and smaller than the distances between the clumps. This galaxy shows that the distribution of globular cluster colours is not necessary well represented by 2 Gaussians.

\subsection{Spatial distributions of the globular clusters}

Figure 3 shows the spatial distribution of the GCCs. The centers of the galaxies are represented by an open square. The distribution of GCs roughly follows the ellipticities of the underlying galaxies. The lack of GCs in the central regions is due to dust, bad fits by GALPHOT (see Sect. 2) or increased noise. Figure 5 shows $V-I$ colours of individual GCCs as a function of radius. These colours as a function of radius are plotted in bins in Fig. 6. Here, triangles, represent the galaxy colour returned by GALPHOT, and circles the average colours of all GCs. For those galaxies for which we can separate the population into red and blue groups (Sect. 4.2) we plot as crosses the mean colours of the red and blue groups respectively.

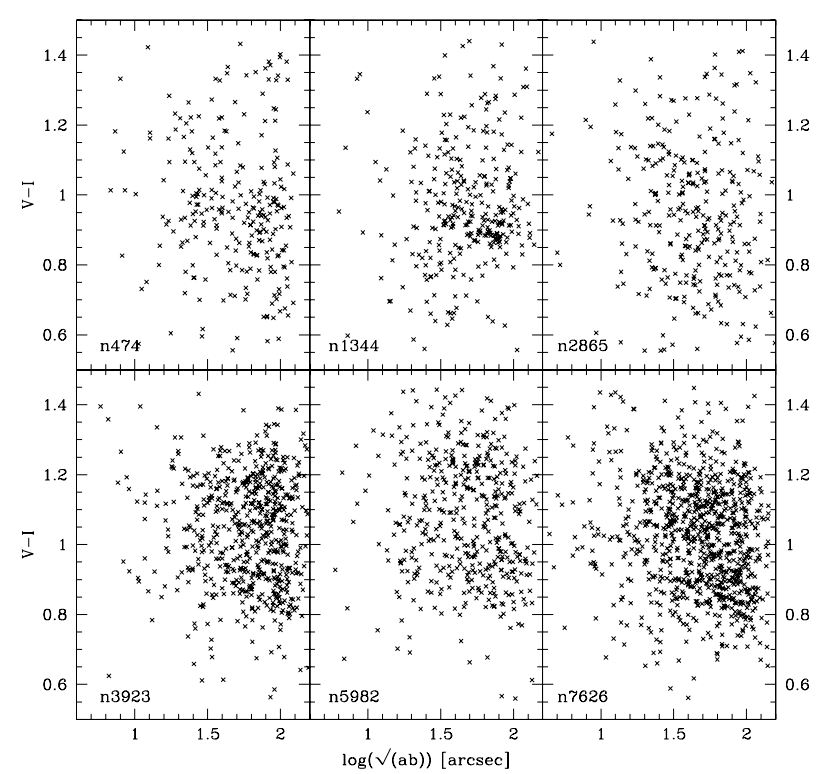

Fig. 5. Globular clusters in $V-I$ (vertical scale) as a function of radius.

Except for NGC 1344, the average colours of all GCs tend to be bluer at large radii, confirming earlier results (Forbes et al. 1996), and reflecting the colour gradients in the stellar halos. Where there is a significant red subgroup the cluster colours in that group match quite well the galaxy colours, except in NGC 3923 where they are bluer, as is the case with NGC 1052 (Forbes et al. 2001a). In NGC 5982 and NGC 7626 the strong blue-ward gradient in the mean cluster colour is caused by a gradient in the relative fractions of the red and blue groups, as illustrated by Fig. 7, where we plot the ratio of the red to the blue population against radius.

\subsection{Globular cluster surface densities}

The GC surface densities were calculated in elliptical annuli; their ellipticity was the average value in the outer regions as determined by GALPHOT. Dusty regions, bad pixels and other bad regions were not used when calculating the effective area of each annulus, from which the surface densities are derived. We accounted for contaminating sources by subtracting a background density, calculated as described in Sect. 3.6. These background densities were between 4.1 to $8.6 \times 10^{-4} \operatorname{arcsec}^{-2}$ for the six galaxies. In Fig. 8 we plot the radial surface density of GCs as a function of radius, the galaxy surface brightness distribution is also shown in this plot. In Figs. 9 and 10, we plot the radial density distribution for the clusters in the red group and blue 


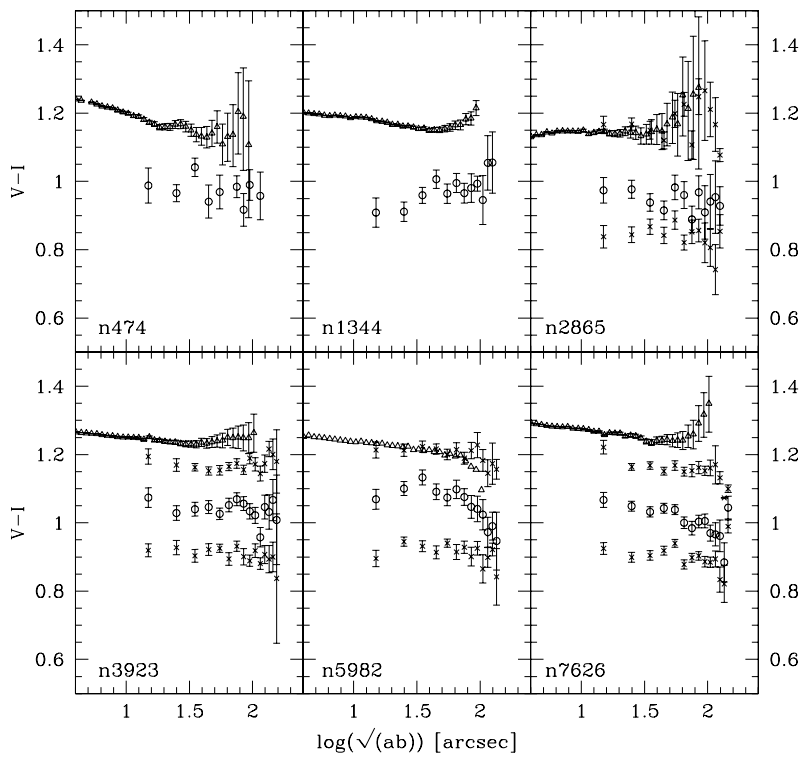

Fig. 6. Average colours of globular clusters (circles: all GCs) and galaxy colour (curve through triangles) as a function of radius. NGC 2865, NGC 3923, NGC 5982 and NGC 7626 also show data points for blue and red groups.

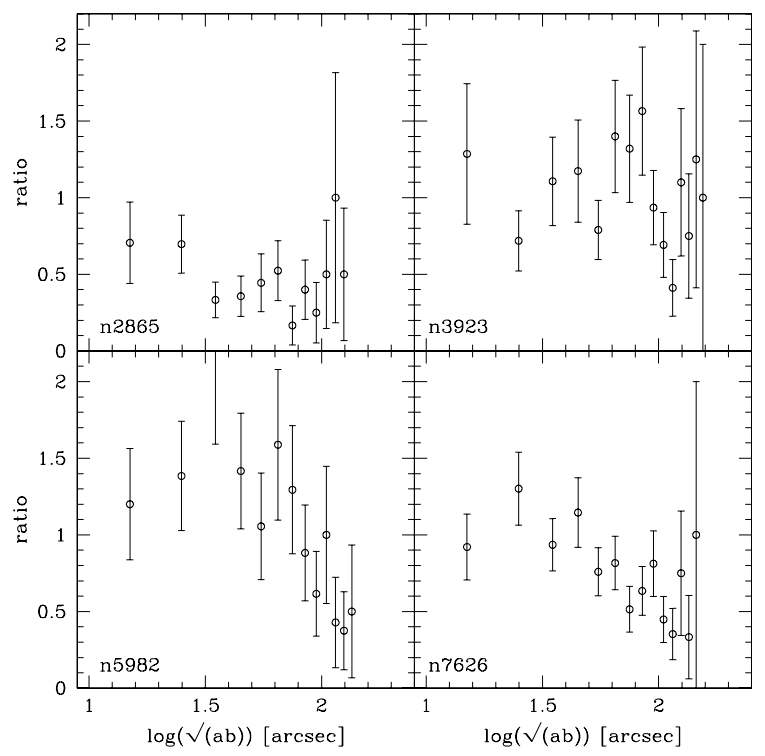

Fig. 7. Ratio of red vs. blue clusters as a function of radius; NGC 5982 and NGC 7626 have strong gradients.

group respectively, for those galaxies with a significant red population.

For all galaxies we note a deficit of clusters in the inner regions, with respect to the background light surface brightness distribution. Possible physical causes of this are discussed in Sect. 7.3, but here we consider the possibility of greater incompleteness in the inner regions causing this deficit. Two effects could contribute to greater incompleteness in the inner regions: confusion due to crowding; and the increased photon noise level in the higher surface brightness regions.

To test the effect of confusion a low density region of $1000 \times$ 1000 pixels, containing 43 clusters, was cut from the residual image of NGC 7626. As in Sect. 3.3, we introduced artificial objects at various number densities, using uniform and powerlaw surface density distributions. After using exactly the same

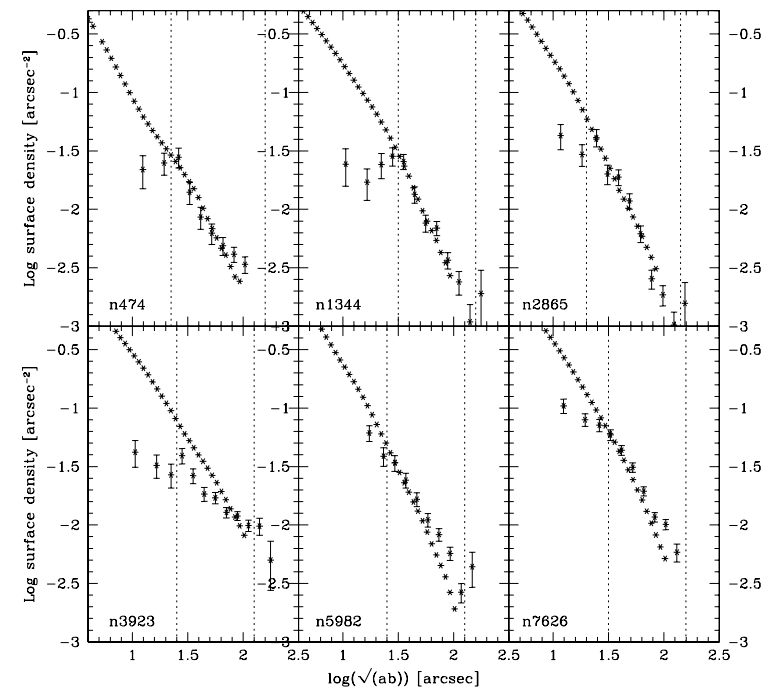

Fig. 8. Surface density of all globular clusters, starlike symbols show galaxy surface brightness (arbitrary scale). The slope of the GC surface density was calculated between the dashed vertical lines.

detection methods and selection criteria as described in Sect. 3.6, we found that we start losing objects due to confusion effects at densities of $0.15 \mathrm{arcsec}^{-2}$, where $5 \%$ are missed. From Fig. 8 we see that even the highest density of our galaxy sample (i.e. NGC 7626) is still below this value.

The effect of the galaxy surface brightness on completeness is due to the increased photon noise in high surface brightness regions. Within the central $10 \operatorname{arcsec}(13.5 \operatorname{arcsec}$ for NGC 5982) this leads to increased incompleteness and we do not plot points within these regions. Outside this, the background photon noise from the galaxy is at most $2 \times$ the background noise, except for NGC 3923 were the photon noise of the inner data point is about $4 \times$ the background photon noise. After again introducing artificial objects and using the same selection criteria as before, we find that this effect is significant only for NGC 3923: the inner two points in Fig. 8 should probably lie somewhat higher.

The radial density distributions of most of the GC systems follow the surface brightness distributions of the galaxy light in the outer parts, but show a deficit at small radii which we argue is not due to incompleteness due to confusion or photon noise from the galaxy. This is typical of ellipticals in general (e.g. Lauer \& Kormendy 1987; Grillmair et al. 1986; Puzia et al. 2004; Forbes et al. 2001a; Schweizer et al. 1996; and Brown et al. 2000). The deficit at the centre is often interpreted as evidence for tidal disruption of clusters passing through the core of the galaxy (Fall \& Rees 1977), but to be effective this process requires the clusters to be on predominantly radial orbits (Grillmair et al. 1986; Ostriker et al. 1989).

NGC 3923 shows a different behaviour, the surface density profile is much shallower than the light profile at all radii (already noted by Zepf et al. 1994). This is typical of the GC systems of some brightest cluster galaxies, e.g. NGC 4874 (Harris et al. 2000). However it is unusual for a galaxy such as NGC 3923, which is the brightest in a small group.

Comparison of Figs. 9 and 10 shows that there are sometimes differences between the surface density profiles of red and blue GCs. To quantify these differences we fit the linear relation $\log (\sigma)=b+\alpha \times r$ by applying a linear weighted least squares fit to the outer points of the density curve. These points were chosen to lie between an inner and outer radius. The inner radius was defined as the point where the flattening stops, estimated by 


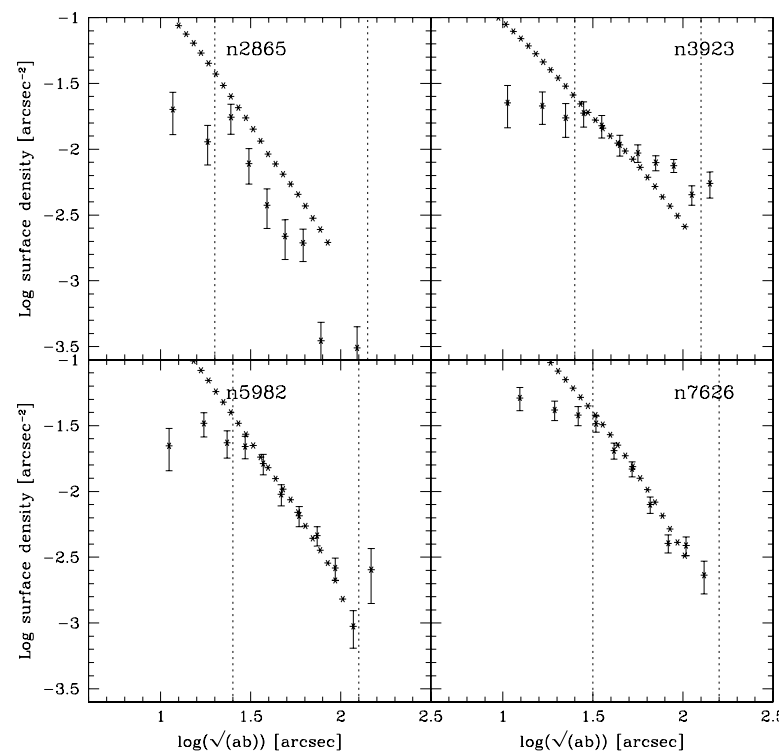

Fig. 9. Surface density of red globular clusters; starlike symbols show the galaxy light (arbitrary scale). The slope of the GC surface density was calculated between the dashed vertical lines.

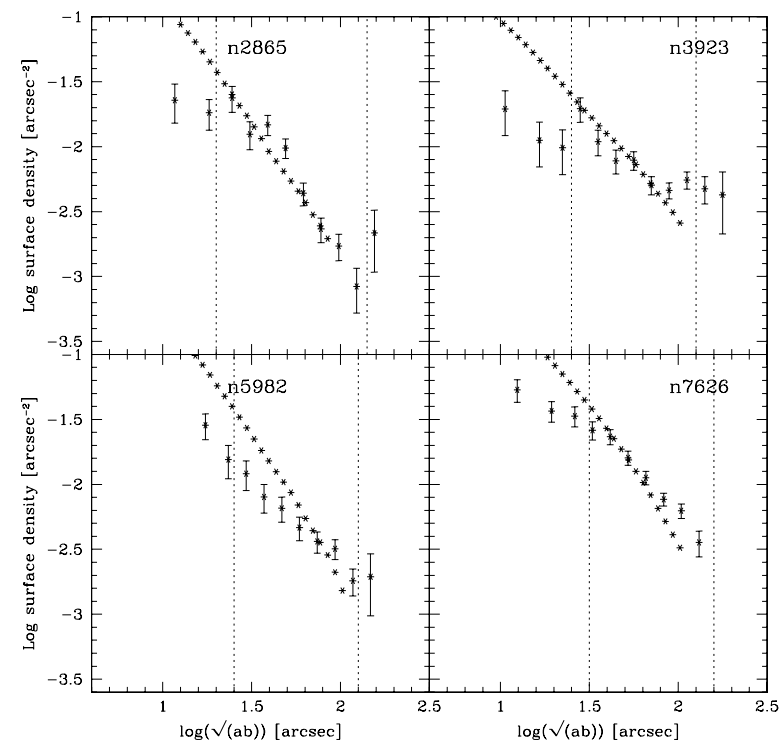

Fig. 10. Surface density of blue globular clusters; starlike symbols show the galaxy light (arbitrary scale). The slope of the GC surface density was calculated between the dashed vertical lines.

eye. The outer radius excludes unreliable data points at larger radii with very low number statistics (usually containing only 1 or 2 objects in the partial elliptical ring located in the very outer corners of the image). We indicate the inner and outer radius in the figures as vertical dotted lines.

Using the same method and radii we also fitted the galaxy light profile. In Cols. 7 to 15 of Table 3 we list the radii used and the results of the fits, including errors on the slopes (we used the standard recipe and error formulae, i.e. Eqs. (15.2.6) and (15.2.9) respectively as listed in Press et al. 1992)

In NGC 7626, the slope of the red GCs is significantly steeper than the slope of the blue GCs. Similar but less significant differences are visible in NGC 2865 and NGC 5982. We checked if these differences are due to the choice of our inner radius by shifting the inner radius one data point to the left as well as to the right; we found no significant change in slope

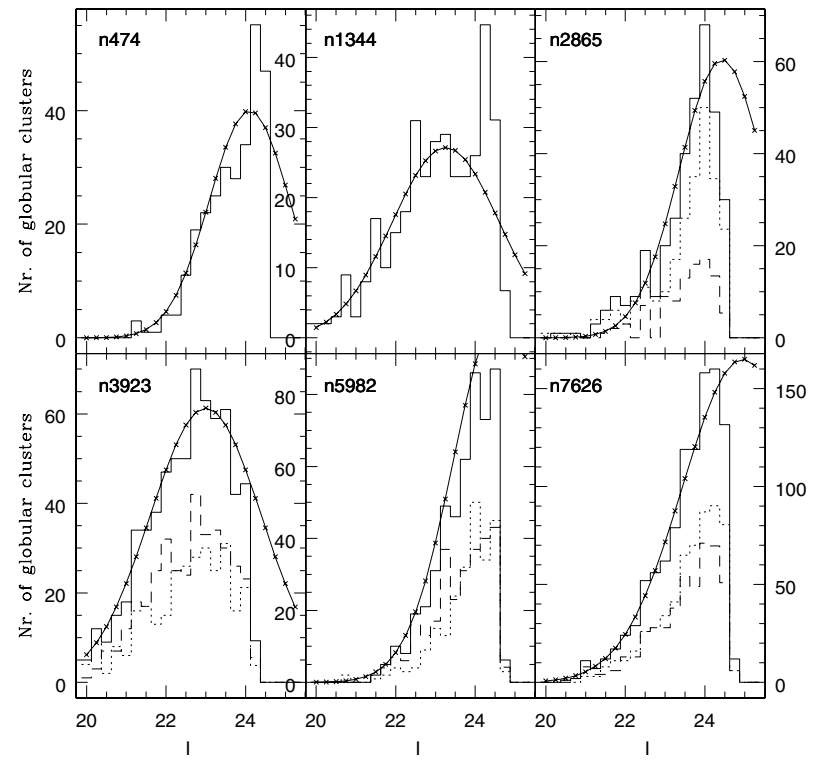

Fig. 11. Globular cluster luminosity functions in the $I$ band. Dashed and dotted histograms represent the GCLF of the red and blue groups respectively. Gaussian fits are also drawn. Only the GCLFs of NGC 3923 and NGC 1344 extend past the TOM.

differences. The slopes calculated for the set of all GCs will be used in Sect. 6, where the specific frequency is calculated.

\section{Globular cluster luminosity function in I}

In this section, the GCLFs in the $I$ band are determined. If the observations are deep enough to cover the absolute turnover magnitude (TOM) of $M_{I}=-8.46 \pm 0.03$ (Kundu \& Whitmore 2001a), the GCLF can be used as a distance estimator. The GCLF can also be used to estimate the number of globular clusters in a system; this number then determines the specific frequency $S_{N}$ (Sect. 6). In those galaxies with a significant red population, we also compare the GCLF of the red and blue samples, which could be different, for instance if the samples have large $\mathrm{M} / \mathrm{L}$ differences (Whitmore et al. 2002).

\subsection{Determination}

We calculated the GCLF in the $I$ band, which is less affected by extinction. The GCLFs were constructed from histograms with a binsize of $0.25 \mathrm{mag}$, approximately twice the photometric error of the faintest objects. The Besançon catalogues were used to correct for contamination by foreground stars. The foreground stars (see Sect. 3.6) were cumulatively subtracted: i.e. if a bin contains only three sources, while there are an expected number of five stars, we subtracted the remaining two stars from the next bin. We only used objects within the $80 \%$ completeness levels and also corrected for incompleteness. The blue and red GCLFs were calculated used the red and blue groups defined in Sect. 4.2. The GCLFs are shown in Fig. 11, where they are divided in blue and red groups, we find no significant differences between the GCLFs of red and blue populations. The peak in the GCLF of NGC 1344 at $I=24.5$ is due to red objects, caused either by photometric errors near the faint limit, or more likely a local excess in the background. 


\subsection{GCLF as a distance estimator for NGC 1344 and NGC 3923}

If the events which formed the shells also formed large numbers of new GCs, then we might find a difference between the TOM of the GCLF, firstly between red and blue populations, and secondly when compared with the canonical value for normal ellipticals of $M_{I_{\text {peak }}}=-8.46$ (Kundu \& Whitmore 2001a). However we only cover this absolute magnitude for the closest two galaxies, NGC 1344 and NGC 3923. Using the IRAF tool NGAUSSFIT we find for NGC $3923 \sigma=1.4 \pm 0.1$, TOM $_{I}=$ $23.04 \pm 0.06$, and, ignoring the spike in the GCLF at faint magnitude, for NGC 1344, $\sigma=1.35 \pm 0.10$, TOM $_{I}=23.26 \pm 0.10$.

Comparing our GCLF distance estimates for NGC 1344 $(m-M=31.72)$ and NGC $3923(m-M=31.50)$ with the SBF distance moduli (from Col. 10 in Table 1: $31.48 \pm 0.30$ and $31.80 \pm 0.28$ respectively), shows that the differences of +0.24 and -0.30 do not exceed the typical errors between these two methods (Richtler 2003). A PNLF distance estimate for NGC 1344 (Teodorescu et al. 2005) with $m-M=31.40 \pm 0.18$ is in excellent agreement with our value. In the remainder we will use our calculated distance moduli for NGC 3923 and NGC 1344.

\subsection{Total numbers of GCs}

A Gaussian fit to the GCLF of the other four galaxies is also necessary to estimate the specific frequency (Sect. 6). Because the magnitude limit is below the TOM of the GCLF, we fit only for $\sigma$ and amplitude, keeping the distance modulus fixed within the error bars (0.26 mag.). Doing this for NGC 5982 and NGC 7626 give $\sigma$ of 1.5 and 1.25 respectively, and good fits. For NGC 474 and NGC 2865 we find $\sigma \sim 1.05$, which is unusually small, and conclude that the calculated $S_{N}$ will be uncertain, and that deeper data are required.

\section{Total number of globular clusters and specific frequencies}

In this section we estimate the total number of clusters and specific frequency $S_{N}$, which is a measure of the number of GCs per unit galaxy luminosity. It is a strong function of galaxy type and environment: late type galaxies and isolated galaxies usually have lower $S_{N}$ than early type and cluster galaxies (Harris 1991). The calculations are straightforward using the results of previous sections. 1981):

The specific frequency is defined by (Harris \& van den Bergh

$S_{N}=N_{\text {tot }} 10^{0.4\left(M_{V}+15\right)}$

where $N_{\text {tot }}$ is the total number of GCs, and $M_{V}$ is the absolute $V$ magnitude of the galaxy, derived from the apparent magnitudes and adopted distances (Table 1).

The total number of GCs is obtained by using:

$N_{\text {tot }}=\frac{N_{\text {Gauss }}}{N_{\text {found }}}\left(N_{\text {missed }}+N_{\text {found }}\right)$

where $N_{\text {found }}$ is the detected number of GCs, corrected for incompleteness, $N_{\text {Gauss }}$ is the expected number of GCs assuming a Gaussian distribution:

$N_{\text {Gauss }}=\frac{1}{\text { binsize }} A \sigma \sqrt{2 \pi}$ with binsize $=0.25$; amplitude and $\sigma$ are from the Gaussian fits from the previous section .

$N_{\text {missed }}$ is the number of GCs expected to be missed due to the fact that the galaxy is much larger than the ACS field of view. This number is calculated by extrapolating and integrating the GC surface density as determined in Sect. 4.4 from the outer points (listed in Col. 7 of Table 3 ), to the point where $\sqrt{a b}$ reaches $50 \mathrm{kpc}$ and $100 \mathrm{kpc}$. Error sources used in the calculation of $S_{N}$ are:

1) a photometric measurement error of $0.15 \mathrm{mag}$;

2) a distance error of $15 \%$. This error is taken from Faber et al. (1989) and represents the scatter in the $D n-\sigma$ distance estimator. Comparing our adopted distances with $D n-\sigma$ distances (see Cols. 11 and 12 in Table 1) shows that this is a reasonable assumption. The distance error was applied to both $N_{\text {miss }}$ and absolute $V$.

3) The errors in the slope of the GC surface density, listed in Col. 13 of Table 3 .

A possible large error is the unknown value of the slope of the GC surface density outside the ACS field of view. We assumed this slope to be equal to the fitted GC density profile in the outer part of the galaxies. Especially for our two most nearby galaxies this assumption is uncertain; these galaxies are only covered by the ACS to $15-17 \mathrm{kpc}$, while we extrapolate until $50 \mathrm{kpc}$ and even $100 \mathrm{kpc}$. Data on the slope in the outer regions of our most distant galaxy, NGC 7626, and surface density profiles in the literature, support this assumption (e.g. Rhode \& Zepf 2004; Zepf et al. 1994; Harris et al. 2000). Finally, for NGC 3923, wide field data (Zepf et al. 1994) show a constant slope to a radius of 5.6 arcmin ( $34 \mathrm{kpc}$ ). Applying a least squares fit to their inner data, covering the ACS fieldsize, gives a slope of $-0.82 \pm 0.34$, comparable to our value $(-0.90 \pm 0.10)$. Their outer data gives a slightly steeper slope of roughly $-1.14 \pm 0.12$, which we used to calculate the missed GCs in Eq. (4).

Assuming our assumption is justified, we adopt the final values for $S_{N}$, which are listed in Cols. 6, 7 (50 kpc) and 8, $9(100 \mathrm{kpc})$ of Table 4 . There sometimes are large variations between $S_{N}$ calculated at $50 \mathrm{kpc}$ and $100 \mathrm{kpc}$, this especially true for NGC 3923 and is due to the shallow slope of the GC density distribution compared with the luminosity distribution. Harris et al. (1998) also noticed such variations and found a ratio $S_{N}^{\text {overall }} / S_{N}^{40}=1.3$, with $S_{N}^{\text {overall }}$ the total $S_{N}$ and $S_{N}^{40}$ the $S_{N}$ within 40kpc. It is important to keep these different definitions of $S_{N}$ in mind if one compares $S_{N}$ with other authors. Some authors calculate local $S_{N}$, others extrapolate to various values ranging between $25 \mathrm{kpc}$ and $200 \mathrm{kpc}$.

Integrated out to $50 \mathrm{kpc}$, we find values of $S_{N}$ typical for isolated elliptical galaxies, except for NGC 3923 and NGC 7626, for which we find $S_{N}=5.6$ and $S_{N}=3.9$ respectively. These values are more typical of cluster ellipticals, indeed NGC 3923 has the highest $S_{N}$ of any isolated elliptical (Zepf et al. 1994). Both of these galaxies are in groups: NGC 3923 is a dominant group galaxy embedded in an X-ray envelope (Buote \& Canizares 1998; Pellegrini 1999); and NGC 7626 is the second brightest member of the Pegasus group and is also detected in X-rays (O' Sullivan et al. 2001).

\section{Discussion}

In this section a short summary of the properties of the shell galaxies is given; a comparison with the GC systems of "normal" 
Table 4. Column 2: detected GCs, Cols. 3, 4: missed GCs + error; Cols. 5, 6: number of GCs + error using the values for the Gaussians determined in Sect. 5.2; Cols. 7, 8: total GCs + error applying Eq. (4); Cols. 9, 10: $S_{N}$ within $50 \mathrm{kpc}$ with error; Cols. 11, 12: $S_{N}$ within $100 \mathrm{kpc}$ with error; Col. 13: ACS range where slope has been determined.

\begin{tabular}{|c|c|c|c|c|c|c|c|c|c|c|c|c|c|}
\hline $\begin{array}{r}\text { Galaxy } \\
(1)\end{array}$ & $\begin{array}{r}N_{\text {found }} \\
\text { (2) }\end{array}$ & $\begin{array}{r}N_{\text {missed }} \\
\text { (3) }\end{array}$ & $\begin{array}{r}\text { Error } \\
(4)\end{array}$ & $\begin{array}{r}N_{\text {Gauss }} \\
\text { (5) }\end{array}$ & $\begin{array}{r}\text { Error } \\
(6)\end{array}$ & $\begin{array}{r}N_{\text {tot }} \\
\text { (7) }\end{array}$ & $\begin{array}{r}\text { Error } \\
(8)\end{array}$ & $\begin{array}{r}S_{N 50} \\
(9)\end{array}$ & $\begin{array}{r}\text { Error } \\
(10)\end{array}$ & $\begin{array}{r}S_{N 100} \\
(11)\end{array}$ & $\begin{array}{r}\text { Error } \\
(12)\end{array}$ & $\begin{array}{r}R_{\text {in }}-R_{\text {out }}(\mathrm{kpc}) \\
(13)\end{array}$ & $\begin{array}{r}M_{V}(\mathrm{mag}) \\
(14)\end{array}$ \\
\hline NGC 474 & 284 & 143 & 29 & 405 & 56 & 609 & 94 & 2.1 & 0.5 & 2.7 & 1.0 & $2.5-25.0$ & -21.2 \\
\hline NGC 1344 & 352 & 93 & 25 & 367 & 28 & 464 & 44 & 1.4 & 0.3 & 1.5 & 0.4 & $3.4-17.0$ & -21.3 \\
\hline NGC 2865 & 350 & 35 & 6 & 647 & 98 & 712 & 108 & 1.6 & 0.4 & 1.7 & 0.4 & $4.1-25.9$ & -21.6 \\
\hline NGC 3923 & 660 & 1254 & 162 & 860 & 67 & 2494 & 286 & 5.6 & 1.3 & 8.3 & 3.6 & $3.1-15.4$ & -21.6 \\
\hline NGC 5982 & 505 & 116 & 18 & 1218 & 161 & 1497 & 202 & 2.6 & 0.6 & 3.0 & 0.9 & $5.1-25.6$ & -21.9 \\
\hline NGC 7626 & 1051 & 141 & 16 & 2498 & 263 & 2833 & 300 & 3.9 & 0.9 & 4.8 & 1.4 & $7.4-37.0$ & -22.2 \\
\hline
\end{tabular}

early type galaxies is made; the data are compared with predictions of the hierarchical merger scenario simulations (Beasley et al. 2002); and possible signs for recent GC formation and ages are discussed.

\subsection{The shell galaxies}

All of our galaxies are located in low density regions; early type galaxies residing in groups and clusters have a much lower probability of exhibiting shells. (Malin \& Carter 1983; Colbert et al. 2001). As well as the presence of the shells, all six galaxies also show visible dust patches and/or lanes, mostly in the central regions. It has been shown that all galaxies, except for NGC 1344, contain a KDC or show otherwise peculiar kinematic behaviour (Hau et al. 1996; Hau et al. 1999; Carter et al. 1998; Emsellem et al. 2004; Balcells \& Carter 1993). NGC 474, NGC 5982 and NGC 7626 may be LINERS (Ho et al. 1997). Except for NGC 474, unresolved X-ray data are available for all galaxies (O' Sullivan et al. 2001) and even a 2D X-ray map for NGC 3923 (Buote \& Canizares 1998; Pellegrini 1999). In this paper we assume that NGC 474 and 1344 are E-type galaxies, although there is evidence in each case that they night be classified as S0 galaxies.

The environmental and other properties of the galaxies are described briefly below (LGG group numbers from Garcia 1993):

$N G C 474$ - brightest galaxy of LGG20 (4 members). The galaxy is connected with the small spiral NGC 470 via a HI tidal bridge (Schiminovich 1997). Often classified as S0; shows some rotation (Emsellem et al. 2004). May be a LINER.

NGC 1344 - located at the outskirts of the Fornax Cluster, $4.9^{\circ}$ from the central cluster galaxy NGC 1399. At this distance the density is comparable to the field galaxy density (Kambas et al. 2000). Sometimes classified as S0; shows rotation within 2 effective radii (Teodorescu et al. 2005).

NGC 2865 - isolated galaxy (Reda et al. 2004), remains of a rotating HI disk (Schiminovich 1995). Hau et al. (1999) found a KDC and evidence for a young (0.4-1.7 Gyr) stellar population; two possible explanations were given: a starburst or a truncation of the star formation.

$N G C 3923$ - brightest galaxy of LGG255 (5 members).

NGC 5982 - brightest galaxy of LGG402 (4 members), may be a LINER.

NGC 7626 - second brightest member of the Pegasus group, LGG473, of at least 15 members. This probable LINER has a radio-jet, directed NE, and a small HI cloud between $1.5^{\prime}$ and 3.0' WSW of the center; the galaxy does not contain any HI tidal features (Hibbard \& Sansom 2003). The core shows orthogonal kinematics to the main body (Balcells \& Carter 1993), with no emission lines, nor signs of nuclear young populations. A dust lane in direction ENE is visible in the inner $15 \mathrm{ACS}$ pixels of the core.

\subsection{Comparison of the GC systems with normal ellipticals}

Our sample is selected on morphological grounds to have undergone a recent minor merger. In this section we investigate the effect of the merger event upon the GC systems. All six galaxies have a peak at $0.94 \pm 0.04$ in their $V-I$ histograms, in common with "normal" ellipticals. In four of our six galaxies we see a distribution of galaxies red-wards of this, this second peak again occurs in the majority of normal ellipticals.

All galaxies show a flattening in the GC density profile near the central regions. This feature is also a generally seen in other GC systems and is attributed to disruption processes (Ostriker et al. 1989; Pesce et al. 1992).

The properties of the GC systems of NGC 3923 and NGC 5982 are very similar. Both galaxies have blue and red populations of comparable size, which peak at roughly the same $V-I$ values near 0.95 and 1.20. Using the colours of the GCCs and assuming that the red peak has solar metallicity, the evolution models of Fritze-v. Alvensleben (2004) (to get the metallicities of the blue clusters) and Fig. 12 from Goudfrooij et al. (2001a) indicate that these systems are old, >5 Gyr, and evolved systems.

NGC 3923 has been extensively studied by Zepf et al. (1995). They already noted the very high $S_{N}$ for a galaxy located in a low density environment, which we confirm. McLaughlin (1999) proposes that high $S_{N}$ galaxies are probably best explained by taking into account the presence of a extended massive X-ray halo. However, NGC 3923 is probably in contradiction with these results. While NGC 3923 has a very shallow GC surface density profile and also contains a X-ray halo (Buote \& Canizares 1998; Pellegrini 1999), its X-ray luminosity is about a factor 10 lower (O' Sullivan et al. 2001; Fukazawa et al. 2006) than the galaxies studied by McLaughlin: M 49, M 87, and NGC 1399.

The colour distributions of NGC 474 and NGC 1344 (Fig. 4) are similar, and appear either to be unimodal, or to have a very low red (metal-rich) component. Although KMM returns a combination of a blue and red peak as a good description of the distribution, there are very few GCs in the red peak. Such colour distributions are very rare in luminous ellipticals (Kundu \& Whitmore 2001a). Peng et al. (2006) show that, in early-type Virgo galaxies, the red peak is much more prominent in the more luminous galaxies. However unimodal, blue, histograms only become common for galaxies fainter than $M_{V}=$ -18 (Figs. 4 and 6 of Peng et al.), whereas the absolute magnitudes of NGC 474 and NGC 1344 are $M_{V}=-21.17$ and -21.07 


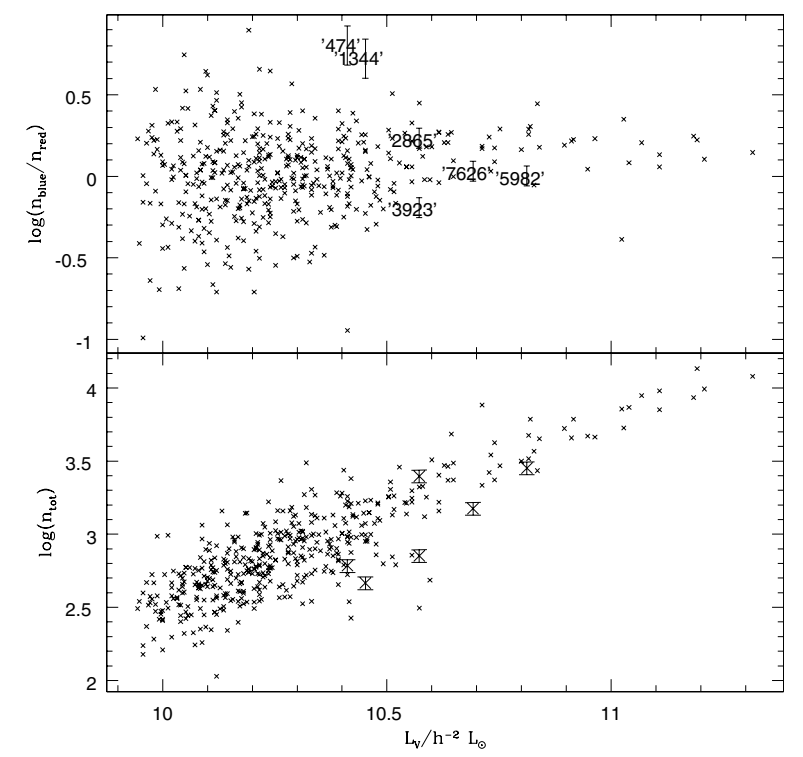

Fig. 12. Top: ratio of blue and red clusters vs. galaxy luminosity. Simulated data (Beasley et al. 2002) and the six shell galaxies (NGC numbers). Bottom: total number of clusters vs. luminosity for simulated data and six shell galaxies (large squares).

respectively. They are however the lowest luminosity and lowest velocity dispersion galaxies in our sample.

Environmental differences in the early history of the galaxy might lead to differences in the GC $(V-I)$ histograms. In the merger model, fewer early mergers would mean less red peak clusters. Fewer early mergers might also cause a galaxy to retain more of its angular momentum, and thus to be an $\mathrm{S} 0$ or a rapidly rotating elliptical. NGC 474 and NGC 1344 both have significant rotation (Emsellem et al. 2004; and Teodorescu et al. 2005, respectively), and both are sometimes classified as S0 galaxies. The most luminous galaxies in the Peng et al. (2006) Virgo sample with unimodal blue histograms are NGC 4660, an elliptical with significant rotation (Emsellem et al. 2004); and NGC 4340, an SB0. However there are counterexamples in the Virgo sample, such as the rotating ellipticals NGC 4564 and NGC 4697 which have red peak dominated colour histograms. Insufficient galaxies have been studied in sufficient depth to analyse any possible correlation between the rotational properties of ellipticals and their GC colour histograms. Kundu \& Whitmore (2001b) find a number of unimodal, blue colour histograms among a sample of S0 galaxies studied with WFPC2 (e.g. NGC 2768), but their sample sizes are small. They do however find that a lower proportion of their sample of SOs are significantly bimodal, than their equivalent sample of ellipticals (Kundu \& Whitmore 2001a).

Finally we compare these objects with some properties predicted by the hierarchical merger model of Beasley et al. (2002). In these semi-analytical simulations, metal-poor GCs are old and formed in cold gas clumps, while the metal-rich are created in time during merger events. Of course, accretion of GCs also takes place during the hierarchical build-up of galaxies. These simulations are able to reproduce the many variations in the colour distributions of GC systems observed in elliptical galaxies. For instance, in Fig. 13 of Beasley et al., a blue-peaked $V-I$ distribution is shown which looks similar to our large single peak $V-I$ distributions of NGC 474 and NGC 1344. His model also roughly reproduces the observed $L_{V}-N_{\text {tot }}$ relation. We plot this relation in the lower part of Fig. 12 together with our six data points. Our systems seem to have somewhat fewer globular clusters than predicted. This is partly explained by the fact that Beasley et al. do not take into account disruption processes, which might reduce the number of clusters by 10\%-20\%. The upper part of the same Figure shows the logarithm of the ratio between the number of blue and red clusters. NGC 474 and NGC 1344, the two points with lowest luminosity, lie somewhat outside the point cloud due to their low number of red clusters.

\subsection{Possible evidence for recent GC formation in NGC 7626 and NGC 2865}

In this section we examine the possibility that the complex $(V-I)$ histograms of NGC 7626 and NGC 2865 provide evidence for recent $\mathrm{GC}$ formation, in addition to the old GC population which gives both the red and blue peaks. In NGC 7626, Fig. 4 shows that the brightest GCs of NGC 7626, with $M_{I} \approx-12.5 \pm 0.2$ and $V-I=1.1$, are more than 1.0 mag over-luminous with respect to the brightest GCs of the other five galaxies (all at about -11.0), and with respect to its own universal blue population. It is the most luminous galaxy in our sample $\left(M_{V}=-22.16\right)$, and as such the GCLF would be expected to extend to the brightest magnitudes, simply because of the larger population at the sparsely populated faint end of the GCLF. We compare the GC population of NGC 7626 with that of NGC 4472 (Rhode \& Zepf 2001), a more luminous galaxy $\left(M_{V}=-22.7\right)$ in the Virgo cluster. NGC 4472 has its brightest clusters in the blue (universal) peak, at $R=19$ (equivalent to $M_{I}=-12.5$ ). The brightest clusters in NGC 7626 are about 0.2 mag brighter and 0.2 mag redder in $(V-I)$ than this, in a less luminous galaxy. This group of bright clusters appears in the GCLF of NGC 7626 (Fig. 11) as a small excess near $I=21$.

We can compare this population with that found by Whitmore et al. (1997) in NGC 3610, a dynamically young elliptical. Their Figure 15 illustrates the evolution of a young, metalrich population compared with an old, metal-poor population. In time, the young, metal rich, GC population will fade in luminosity and will become redder. After three Gyr, this population has become redder than the old population but still has several GCs which are brighter than the brightest old metal poor GC. This is exactly what is visible in our colour magnitude diagram. Whitmore et al. quantify the age differences using the $\Delta(V-I)$ vs. $\Delta V_{10}$ diagram (see their Fig. 18). This diagram represents an age sequence by plotting vertically the difference in $V-I$ between the peaks and horizontally the magnitude difference between the 10th brightest globular cluster in the young and old populations. In NGC $7626 \Delta V_{10}$ is extremely difficult to estimate, because the young population is dominated in number not just by the old metal-poor population, but by an old, red, metal-rich population as well. Replacing $\Delta V_{10}$ by an estimate of the difference between the magnitude of the brightest clusters and the brightest in the blue peak, we estimate a very tentative merger age of 2-5 Gyr. We conclude that NGC 7626 appears to have some young GCs, probably created in a recent (2-5 Gyr old) minor merger. These young GCs are superimposed upon a much richer, bimodal, old cluster population.

For all galaxies with a substantial red population, we find significantly steeper slopes for the red clusters. This is reflected in Fig. 7, where gradients are seen in the ratio of red to blue clusters as a function of position: the red GCs are more centrally concentrated than the blue GCs. Similar effects are also seen in other early type galaxies (NGC 1407: Forbes et al. 2006; NGC 4649: Forbes et al. 2001a; NGC 1399: Dirsch et al. 2005; NGC 4636: Dirsch et al. 2005; and others). 
Table 5. Age estimates in Gyr. Column 2: SSP ages from Denicoló et al. (2005) except $\uparrow$ : Hau et al. (1999) and $\ddagger$ Kuntschner et al. 2002; Col. 3: ages using fine-structure index $\Sigma$ method (Schweizer \& Seitzer 1992); Col. 4: Old (NP) and Young (YP) systems together with their $\Sigma_{2}$ index according to Michard \& Prigniel (2004); Col. 5: dynamical ages of type I shell systems using Nulsen (1989); Col. 6: approximate age indications from $\mathrm{GC}(V-I)$ distribution (this paper).

\begin{tabular}{rrrrrr}
\hline \hline $\begin{array}{r}\text { Galaxy } \\
(1)\end{array}$ & $\begin{array}{r}\text { SSP } \\
(2)\end{array}$ & $\begin{array}{r}\Sigma \text {-method } \\
(3)\end{array}$ & $\begin{array}{r}\text { YP/NP }\left(\Sigma_{2} \text { index }\right) \\
(4)\end{array}$ & $\begin{array}{r}\text { Shell dynamical } \\
(5)\end{array}$ & $\begin{array}{r}\text { GCs } \\
(6)\end{array}$ \\
\hline NGC 474 & $7.3_{-2.4}^{+2.0}$ & $5.4 \pm 1.9$ & - & - & Old system \\
NGC 1344 & $4.0 \pm 1.0 \ddagger$ & - & NP $(5.5)$ & 0.5 & Old system \\
NGC 2865 & Old plus $0.1-1.7 \dagger$ & - & YP $(10.6)$ & - & Old plus 0.5-1 \\
NGC 3923 & $2.6_{-0.6}^{+0.5}$ & - & NP $(10.3)$ & $0.8-1.3$ & Old system \\
NGC 5982 & $12.3_{-2.0}^{+1.9}$ & $6.8 \pm 1.5$ & YP $(6.8)$ & 0.2 & Old system \\
NGC 7626 & $13.9_{-2.4}^{+4.9}$ & $8.0 \pm 1.3$ & NP $(1.4)$ & - & Old plus 2-5 \\
\hline
\end{tabular}

Since the converse situation is never or rarely seen, this must reflect some important difference between blue and red clusters. This difference will be related to different formation processes, combined with disruption, which will affect populations differently depending upon their orbital structure. Less radial orbits for the red GCs might explain this, but without kinematic data on large samples of GCs nothing conclusive can be said about the cause of these differences.

NGC 2865 shows all signs of a recent merger event: a very luminous shell and a KDC with evidence of a recent, 0.4-1.7 Gyr, starburst (Hau et al. 1999), and an HI disk (Schiminovich 1995).

The $(V-I)$ histogram of the GCs of NGC 2865 (Fig. 4) is more complex than a simple bimodal structure, with a population of very blue, not particularly luminous, GCs near $(V-I)=0.7$. The colour of this population is consistent with an age in the range 0.5-1 Gyr (Whitmore et al. 1997), consistent with the nuclear starburst age of 0.4-1.7 Gyr (Hau et al. 1999), but the luminosity of the brightest clusters is much fainter than predicted. This could be attributed to the small number of clusters in the young population, or else to different physical conditions imposing a different Globular Cluster Mass Function.

An alternative hypothesis is that the structure in the colourmagnitude diagrams of the GC systems of NGC 2865 and NGC 7626 is entirely due to metallicity variations. In the case of NGC 2865 this would require a population of old clusters with $(V-I) \sim 0.7$, which is too blue for an old population at any metallicity (e.g. Lee \& Carney 2002). In NGC 7626 an old population of intermediate metallicity could fill in the dip in the CM diagram, but it would have to have a very unusual luminosity function to produce the numbers of bright clusters that we see.

\subsection{Ages and minor mergers}

In this section we compare ages derived for the stellar population of the galaxy; for the shell-forming merger events; and for the young GCs that we argue are present in NGC 2865 and NGC 7626. Stellar ages derived by comparison with Single Stellar Population (SSP) models are uncertain for a number of reasons. First, galaxies are clearly not SSPs, and merger remnants in particular will have at least three episodes of star formation, corresponding to the two progenitors and to the merger induced star formation event. Second, the degeneracy between age and metallicity, combined with uncertainty in isochrone models, and assumptions made about other parameters of the stellar population, such as the mass function, render SSP ages very uncertain (Poggianti et al. 2001). Third SSP ages are derived from nuclear spectra only, which can be unrepresentative of the galaxy as a whole (e.g. Proctor et al. 2005). Nevertheless SSP ages have been computed for four of our sample by Denicolo et al. (2005), for NGC 1344 by Kuntschner et al. (2002), and for NGC 2865 a starburst age has been derived by Hau et al. (1999), and these are listed in Table 5.

Schweizer \& Seitzer (1992) determine a Fine Structure Index, based upon the galaxy morphology, and derive an empirical correlation between this and the stellar age. The Fine Structure Parameter measures the age since the galaxy as a structure was built up, and this is different from the ages of the stars in the galaxy. However it determines a combination of the age of and the magnitude of a merger event, so there is a degeneracy here as well. Schweizer \& Seitzer quote Fine Structure ages for three of our sample which are listed in Col. 3 of Table 5. A similar approach was carried out by Michard \& Prugniel (2004). They divided their peculiar elliptical galaxy sample into a normal (NP), reddish, sample, with no signs of a young stellar population and a bluish sample (YP) with evidence for a younger stellar population mixed with an old one. They list five of our galaxies and their results are listed in the fourth column of Table 5.

Dynamical ages for phase-wrapped, type I shell systems such as NGC 1344, NGC 3923 and NGC 5982 can be derived from shell radii and spacings of the outer shells, where dynamical friction and tidal effects are unimportant in determining the particle distribution (Nulsen 1989). We used Nulsen's Eq. (88) to derive the dynamical ages. Mass estimates were taken from the literature (NGC 1344: Teodorescu et al. 2005; NGC 3923: Fukazawa et al. 2006; fundamental plane masses: Eq. (7) of van Dokkum \& Stanford 2003). The calculated ages are shown in Table 5 and are of the order of several 100 Myr. It is clear that these young ages are not reflected in the GC populations for these galaxies, implying that the shell forming event did not in these cases give rise to a substantial GC population.

Our sample consists of minor merger remnants, and only if it is possible to separate the old and merger related populations, as in the case of NGC 2865, can we see a correspondence between the different age estimators. More detailed studies of the stellar populations of all of the other galaxies would be valuable, as would better theoretical estimates of the dynamical ages of the more complicated Prieur (1990) type II and III shell systems.

\section{Conclusions}

The properties of the globular cluster systems of six shell galaxies were analysed. For NGC 2865 and NGC 7626 we observe anomalous features in the $(V-I)$ histograms, in addition to the bimodal structure which is normal for ellipticals. The features represent excesses at intermediate colour in NGC 7626, and at very blue colours in NGC 2865, consistent with a small 
population of GCs, of age 2-5 Gyr and 0.5-1 Gyr respectively, possibly formed in the merger event which created the shells. In each case the young population is dominated by the much larger, old, bimodal distribution.

The data for two galaxies (NGC 1344 and NGC 3923) allow the determination of their globular cluster luminosity function distances. Fitting Gaussians to their GCLF give distances moduli of 31.72 and 31.50 for NGC 1344 and NGC 3923 respectively.

The properties of NGC 3923 and NGC 5982 are very similar. Their bimodal $V-I$ distributions and radial density profiles of blue and red clusters are typical for old GC systems in ellipticals. NGC 474 and NGC 1344 show one single blue peak and very shallow red peaks in their $V-I$ histograms. These properties are unusual for bright ellipticals (Kundu \& Whitmore 2001a, Peng et al. 2006) and may indicate less early mergers in their formation history.

NGC 3923 and NGC 7626 have higher specific frequencies (respectively 5.6 and 3.9 within $50 \mathrm{kpc}$ ) than normal for galaxies located in a low density environment. The luminosity of the $\mathrm{X}$-ray halo detected in the former galaxy is probably not sufficient to explain its high $S_{N}$ as proposed by McLaughlin (1999). The $S_{N}$ of the other galaxies have values of around 2 and are typical for galaxies located in a low density environment.

Although for some of these six galaxies new GCs may have been formed recently, the general properties (like $V-I$ distributions and flattening of GC density profile) of the globular cluster systems of these shell galaxies do not deviate systematically from "normal" elliptical galaxies.

In a future paper (Sikkema et al., in preparation) we will investigate the morphology and stellar populations of the diffuse galaxy, in particular in the shells.

Acknowledgements. We thank an anonymous referee for helpful comments. The ACS observations were made with the NASA/ESA Hubble Space Telescope, operated by the Space Telescope Science Institute, which is operated by the Association of Universities for Research in Astronomy under NASA contract NAS 5-26555. These observations are associated with programme GO9399.

\section{References}

Ashman, K. A., \& Zepf, S. E. 1992, 384, 50

Ashman, K. A., Bird, C. M., \& Zepf, S. E. 1994, AJ, 108, 2348

Balcells, M., \& Carter, D. 1993, A\&A, 279, 376

Battinelli, P., \& Capuzzo-Dolcetta, R. 1989, ApJ, 347, 794

Beasley, M. A., Baugh, C. M., Forbes, D. A., et al. 2002, MNRAS, 333, 383

van den Bergh, S. 1981, AJ, 86, 1464

van den Bergh, S. 1991, IAUS, 148, 161

van den Bergh, S. 1994, AJ, 108, 2145

Bertin, E., \& Arnouts, S. 1996, A\&AS, 117, 393

Brown, R. J. N., Forbes, D. A., Kissler-Patig, M., \& Brodie, J. P. 2000, MNRAS, 317,406

Buote, D. A., \& Canizares, C. R. 1998, MNRAS, 298, 811

Capuzzo-Dolcetta, R., \& Tesseri, A. 1997, MNRAS, 292, 808

Capuzzo-Dolcetta, R., \& Donnarumma, I. 2001, MNRAS, 328, 645

Carter, D. 1978, MNRAS, 182, 797

Carter, D., Thomson, R. C., \& Hau, G. K. T. 1998, MNRAS, 294, 182

Côté, P., Marzke, R. O., \& West, M. J. 1998, ApJ, 501, 554

Cohen, J. G., Blakeslee, J. P., \& Ryzhov, A. 1998, ApJ, 496, 808

Cohen, J. G., Blakeslee, J. P., \& Côté, P. 2003, ApJ, 592, 866

Colbert, J. W., Mulchaey, J. S., \& Zabludoff, A. I. 2001, AJ, 121, 808

Denicoló, G., Terlevich, R., Terlevich, E., et al. 2005, MNRAS, 358, 813

Dirsch, B., Richtler, T., Geisler, D., et al. 2003, AJ, 125, 1908

Dirsch, B., Schuberth, Y., \& Richtler, T. 2005, A\&A, 433, 43

de Vaucouleurs, G. 1948, Ann. Ap., 11, 247

van Dokkum, P. G. 2001, PASP, 113, 1420

van Dokkum, P. G., \& Stanford, S. A. 2003, ApJ, 585, 78

van Dokkum, P. G., Franx, M., Förster Schreiber, N. M., et al. 2004, ApJ, 611, 703
Ellis, R. S., Smail, I., Dressler, A., et al. 1997, ApJ, 483, 582

Emsellem, E., Cappellari, M., Peletier, R. F., et al. 2004, MNRAS, 352, 721

Faber, S. M., Wegner, G., Burstein, D., et al. 1989, ApJS, 69, 763

Fall, S. M., \& Rees, M. J. 1977, MNRAS, 181, 37P

Fall, S. M., \& Zhang, Q. 2001, ApJ, 561, 751

Forbes, D. A., \& Forte, J. C. 2001, MNRAS, 322, 257

Forbes, D. A., Franx, M., Illingworth, G. D., \& Carollo, C. M. 1996, ApJ, 467, 126

Forbes, D. A, Brodie, J. P., \& Grillmair, C. J. 1997, AJ, 113, 1652

Forbes, D. A., Georgakakis, A. E., \& Brodie, J. P. 2001a, MNRAS, 325, 1431

Forbes, D. A., Beasley, M. A., Brodie, J. P., \& Kissler-Patig, M. 2001b, ApJ, 563, L143

Forbes, D. A., Strader, J., \& Brodie, J. P. 2004a, AJ, 127, 3394

Forbes, D. A., Faifer, F. R., Forte, J. C., et al. 2004b, MNRAS, 355, 608

Forbes, D. A., Sanchez-Blazquez, P., Phan, A., et al. 2006 [arXiv: astro-ph/0510599]

Frenk, C. S., \& Fall, S. M. 1982, MNRAS, 199, 565

Fukazawa, Y., Botoya-Nonesa, J. G., Pu, J., Ohto, A., \& Kawano, N. 2006, ApJ, 636,698

Fritze-v. Alvensleben, U. 2004, A\&A, 414, 515

Garcia, A. M. 1993, A\&AS, 100, 47

Gascoigne, S. C. B., \& Kron, G. E. 1952, PASP, 64, 196

Gebhardt, K., \& Kissler-Patig, M. 1999, AJ, 118, 1526

Girardi, L., Chiosi, C., Bertelli, G., \& Bressan, A. 1995, A\&A, 198, 87

Gnedin, O. Y., \& Ostriker, J. P. 1997, ApJ, 474, 223

Goudfrooij, P., Mack, J., Kissler-Patig, M., et al. 2001a, MNRAS, 322, 643

Goudfrooij, P., Alonso, M. V., Maraston, C., \& Minniti, D. 2001b, MNRAS, 328, 237

Goudfrooij, P., Gilmore, D., Whitmore, B. C., et al. 2004, ApJ, 613, L121

Grillmair, C., Pritchet, C., \& van den Bergh, S. 1986, AJ, 91, 1328

Harris, W. E. 1991, ARA\&A, 29, 543

Harris, W. E., \& van den Bergh, S. 1981, AJ, 429, 177

Harris, W. E., Harris, G., \& McLaughlin, D. E. 1998, AJ, 115, 1801

Harris, W. E., Kavelaars, J. J. Hanes, D. A., Hesser, J. E., \& Pritchet, C. J. 2000, ApJ, 533, 137

Hau, G. K. T., Balcells, M., \& Carter, D. 1996, IAUS, 171, 388

Hau, G. K. T., Carter, D., \& Balcells, M. 1999, MNRAS, 306, 437

Hernquist, L., \& Quinn, P. J. 1987, ApJ, 312, 1

Hibbard, J. E., \& Sansom, A. E. 2003, AJ, 125, 667

Ho, L. C., Filippenko, A. V., \& Sargent, W. L. 1997, ApJS, 112, 315

Ibata, R., Gilmore, G., \& Irwin, M. 1995, MNRAS, 277, 781

Jørgensen, I., Franx, M., \& Kjaergaard, P. 1992, A\&AS, 95, 489

Kambas, A., Davies, J. I., Smith, R. M., Bianchi, S., \& Haynes, J. A. 2000, AJ, 120,1316

Kundu, A., \& Whitmore, B. C. 2001a, AJ, 121, 2950

Kundu, A., \& Whitmore, B. C. 2001b, AJ, 122, 1251

Kuntschner, H., Smith, R. J., Colless, M., et al. 2002, MNRAS, 337, 172

Lauer, T. R., \& Kormendy, J. 1987, ApJ, 303, L1

Lee, J.-W., \& Carney, B. W. 2002, AJ, 123, L3305

Malin, D. F., Carter, D. 1980, Nature, 285, 643

Malin, D. F., \& Carter, D. 1983, ApJ, 274, 534

McLaughlin, D. E. 1999, AJ, 117, 2398

Michard, R., \& Prugniel, P. 2004, A\&A, 423, 833

Miller, B. W., Whitmore, B. C., Schweizer, F., \& Fall, S. M. 1997, AJ, 114, 2381

Nulsen, P. E. J. 1989, ApJ, 346, 690

Ostriker, J. P., Binney, J., \& Saha, P. 1989, MNRAS, 241, 849

O' Sullivan, E., Forbes, D. A., \& Ponman, T. J. 2001, MNRAS, 328, 461

Peebles, P. J. E. 2002, in A new Era in Cosmology, ed. N. Metcalfe, \& T. Shanks (San Francisco: ASP), ASP Conf. Proc., 283, 351

Pellegrini, S. 1999, A\&A, 343, 23

Peng, E. W., Jordán, A., Côté, P., et al. 2006, ApJ, in press [arXiv: astro-ph/0509654]

Pesce, E., Capuzzo-Dolcetta, R., \& Vietri, M. 1992, MNRAS, 254, 466

Poggianti, B. M., Bridges, T. J., Mobasher, B., et al. 2001, ApJ, 562, 689

Press, W. H., Teukolsky, S. A., Vetterling, W. T., \& Flannery, B. P. 1992, Numerical Recipes in C, 2nd edn. (Cambridge: Cambridge Univ. Press)

Prieur, J.-L. 1990, in Dynamics and Interactions of Galaxies, ed. R. Wielen (Berlin: Springer-Verlag), 72

Proctor, R. N., Forbes, D. A., Forestell, A., \& Gebhardt, K. 2005, MNRAS, 362, 857

Puzia, T. H., Kissler-Patig, M., Brodie, J. P., \& Huchra, J. P. 1999, AJ, 118, 2734

Puzia, T. H., Kissler-Patig, M., Thomas, D., et al. 2004, A\&A, 415, 123

Quinn, P. J. 1984, ApJ, 279, 596

Reda, F. M., Forbes, D. A., Beasley, M. A., et al. 2004, MNRAS, 354, 851

Rhode, K. L., \& Zepf, S. E. 2001, AJ, 121, 210

Rhode, K. L., \& Zepf, S. E. 2004, AJ, 127, 302

Richtler, T. 2003, in Stellar Candles for the Extragalactic Distance Scale, ed. D. Alloin, \& W. Gieren, Lecture Notes Phys., 635, 281 
Roberts, M. S., Hogg, D. E., Bregman, J. N., Forman, W. R., \& Jones, C. 1991, ApJS, 75, 751

Robin, A. C., Reylé, C., Derrière, S., et al. 2003, A\&AS, 409, 523

Schlegel, D. J., Finkbeiner, D. P., \& Davis, M. 1998, ApJ, 500, 525

Schiminovich, D., van Gorkom, J. H., van de Hulst, J. M., \& Malin, D. F. 1995, ApJ, 444, L77

Schiminovich, D., van Gorkom, J. H., \& van de Hulst, J. M. 1997, IAUS, 186

Schweizer, F. 1987, in Nearly normal galaxies, from the Planck time to the present, ed. S. M. Faber (New York: Springer-Verlag), 18

Schweizer, F., \& Seitzer, P. 1992, AJ, 104, 1039

Schweizer, F., Miller, B. W., Whitmore, B. C., \& Fall, S. M. 1996, AJ, 112, 1839

Sirianni, M., Jee, M. J., Benítez, N., et al. 2005, PASP, 117, 1049

Strader, J., Brodie, J. P., \& Forbes, D. A. 2004, AJ, 127, 3431

Strader, J., Brodie, J. P., Cenarro, A. J., et al. 2005, AJ, 130, 1315

Teodorescu, A. M., Méndez, R. H., Saglia, R. P., et al. 2005, 635, 290

Tonry, J. L., Dressler, A., Blakeslee, J. P., et al. 2001, ApJ, 546, 681
Toomre, A. 1977, in Evolution of Galaxies and Stellar Populations, ed. B. M. Tinsley, \& R. B. Larson (New Haven: Yale University Observatory), 401 Tremaine, S. D., Ostriker, J. P., \& Spitzer, L. S. 1975, ApJ, 196, 407 Valentijn, E. A., \& Kuijken, K. 2004, in Toward an International Virtual Observatory, ed. P. J. Quinn, \& K. M. Gorski (Springer), 19

Whitmore, B. C., \& Schweizer, F. 1995, AJ, 109, 960

Whitmore, B. C., Sparks, W. B., Lucas, R. A., et al. 1995, ApJ, 454, L73

Whitmore, B. C., Miller, B. W., Schweizer, F., \& Fall, S. M. 1997, AJ, 114, 1797

Whitmore, B. C., Zhang, Q., Leitherer, C., et al. 1999, AJ, 118, 1551

Whitmore, B. C., Schweizer, F., Kundu, A., \& Miller, B. W. 2002, AJ, 124, 147

Williams, R. E., Blacker, B., Dickinson, M., et al. 1996, AJ, 112, 1335

Yoon, S.-J., Yi, S. K., \& Lee, Y.-W. 2006, Science, 311, 1129

Zepf, S. E., \& Ashman, K. A. 1993, MNRAS, 264, 611

Zepf, S. E., Geisler, D., \& Ashman, K. M. 1994, ApJ, 435, L117

Zepf, S. E., Ashman, K. M., \& Geisler, D. 1995, ApJ, 443, 570 